MATHEMATICS OF COMPUTATION

Volume 79, Number 269, January 2010, Pages 95-124

S 0025-5718(09)02293-5

Article electronically published on July 29, 2009

\title{
A NUMERICAL METHOD FOR FRACTAL CONSERVATION LAWS
}

\author{
JÉRÔME DRONIOU
}

\begin{abstract}
We consider a fractal scalar conservation law, that is to say, a conservation law modified by a fractional power of the Laplace operator, and we propose a numerical method to approximate its solutions. We make a theoretical study of the method, proving in the case of an initial data belonging to $L^{\infty} \cap B V$ that the approximate solutions converge in $L^{\infty}$ weak-* and in $L^{p}$ strong for $p<\infty$, and we give numerical results showing the efficiency of the scheme and illustrating qualitative properties of the solution to the fractal conservation law.
\end{abstract}

\section{INTRODUCTION}

Partial differential equations involving non-local operators are used in several models, from mathematical finance [23] to dislocation dynamics [5] to gas detonation $[13$ and anomalous diffusion in semiconductor growth [26]. We consider in this paper the following model of a non-local scalar conservation law, which appears in particular in the last two references:

$$
\begin{cases}\partial_{t} u(t, x)+\partial_{x}(f(u(t, x)))+g[u(t, \cdot)](x)=0 & t>0, x \in \mathbb{R} \\ u(0, x)=u_{0}(x) & x \in \mathbb{R}\end{cases}
$$

where $f: \mathbb{R} \mapsto \mathbb{R}$ is locally Lipschitz continuous, $u_{0} \in L^{\infty}(\mathbb{R}) \cap B V(\mathbb{R})$ and $g$ is a fractional power of order $\lambda / 2$ of the Laplacian, with $\lambda \in] 0,2[$. The natural definition of $g$ can be written via the Fourier transform $g[\phi]=\mathcal{F}^{-1}\left(|\cdot|{ }^{\lambda} \mathcal{F}(\phi)\right)$, but it will be more useful in the sequel to consider the following formula (see [15): for all $r>0$ and all $\phi \in \mathcal{S}(\mathbb{R})$,

$$
\begin{aligned}
g[\phi](x)= & -c(\lambda) \int_{|z| \leq r} \frac{\phi(x+z)-\phi(x)-\phi^{\prime}(x) z}{|z|^{1+\lambda}} d z \\
& -c(\lambda) \int_{|z|>r} \frac{\phi(x+z)-\phi(x)}{|z|^{1+\lambda}} d z \\
= & g_{\lambda, r}[\phi](x)+g_{0, r}[\phi](x),
\end{aligned}
$$

where $c(\lambda)=\frac{\lambda \Gamma\left(\frac{1+\lambda}{2}\right)}{2 \sqrt{\pi} \pi^{\lambda} \Gamma\left(1-\frac{\lambda}{2}\right)}$ with $\Gamma$ the Euler function (this value of $c(\lambda)$ corresponds to the convention $\mathcal{F}(\phi)(\xi)=\int_{\mathbb{R}} e^{-2 i \pi x \xi} \phi(x) d x$, and gives in fact $g=$ $\left.(2 \pi)^{-\lambda}(-\Delta)^{\lambda / 2}\right)$; the notation $g_{\lambda, r}$ and $g_{0, r}$ refer to the order of each term: the

Received by the editor April 25, 2009 and, in revised form, March 23, 2009.

2000 Mathematics Subject Classification. Primary 65M12, 35L65, 35S10, 45K05.

Key words and phrases. Conservation laws, Lévy operator, fractal operator, integral operator, numerical scheme, proof of convergence, numerical results.

(C)2009 American Mathematical Society Reverts to public domain 28 years from publication 
first term is of order $\lambda$ (the singularity of the weight in the integral sign necessitates some regularity on $\phi$, of the kind $\left.\phi(x+z)-\phi(x)-\phi^{\prime}(x) z=o\left(|z|^{\lambda}\right)\right)$, whereas the second term can be applied to any bounded non-regular $\phi$ and is therefore of order 0 .

There are several theoretical studies and results regarding such equations. To our knowledge, [6] presents some of the first results on these problems, mainly with $f(s)=s^{2}$ (or other powers) and an $H^{s}$ or Morrey framework, studying in particular traveling wave or self-similar solutions; more on self-similar solutions, as well as time decay estimates, can be found in 8. In the framework of bounded solutions, classical for pure scalar conservation laws, the existence and uniqueness of a regular solution if $\lambda>1$ has been proved in 14. If $\lambda \leq 1$, the solution is not smooth in general (see [3]) and obtaining general existence and uniqueness results for (1.1) requires using an appropriate notion of entropy solution, introduced and studied in [1; this notion, which is constructed from the classical notion for scalar conservation laws [18], relies on the formula (1.2).

Numerical studies of non-local operators in first-order PDE seem more scarce. A scheme for a Hamilton-Jacobi equation modelling dislocation dynamics and involving a non-local zero-order velocity is studied in [16] (see also the references therein). Closer to the framework of scalar conservation laws, 22 studies an equation modelling the formation and movement of dunes, which is (1.1) with an additional term $-\Delta u$ and $g$ given by the opposite of (1.2) with $\lambda=4 / 3$ and an integral sign only on $\mathbb{R}^{-}$(the non-local operator is therefore a lower-order term in the PDE); besides theoretical results on the solution to this non-monotone equation, numerical results are obtained using a simple finite difference scheme (explicit and with centered discretizations), the study of which remains to be done. Regarding numerical approximations of (1.1) itself, to our best knowledge the only existing results are those based on the probabilistic interpretation of this equation (fractal conservation laws can be, as the classical heat equation, linked with a stochastic differential equation): 20] and 24] use this interpretation to construct and study, in the case $\lambda>1$, a numerical method for 1.11); however, in order to avoid having a too noisy approximation of the solution, the probabilistic method must be applied on the equation on $\partial_{x} u$ obtained by derivating (1.1) and expressing $u$ as the integral sum of its derivative (the local non-linearity in (1.1) is thus transformed into a non-local non-linearity); this technique is easy to implement in dimension 1, but its adaptation to the multi-dimensional case is less straightforward. (The derived equation becomes a system in which, in order to reconstruct $u$ from its derivatives, one has to introduce a convolution product with the derivative of the fundamental solution to the Laplace equation; see [19] for $g=-\Delta$. This derivative is however a singular function and therefore does not seem easy to use, in a numerical method, without introducing additional errors.)

In this paper, we propose and study a numerical method to directly approximate the solution to (1.1) for any $\lambda \in] 0,2[$. The scheme is based on classical techniques of numerical approximation of scalar conservation laws and diffusion equations (monotone fluxes, semi-implicit scheme, etc.) and therefore, though we present it for (1.1) for the sake of legibility, its adaptation to multi-dimensional equations with heterogeneous fluxes and source terms ( $\operatorname{such}$ as $\partial_{t} u+\operatorname{div}(f(t, x, u))+g[u(t, \cdot)](x)=$ $h(t, x, u))$ is straightforward. This approach also allows us to obtain a stable and 
robust method, valid for any $\lambda \in] 0,2[$ and which preserves the qualitative properties of the solution, such as the symmetry, the maximum principle (the solution takes its values between the upper and lower bounds of the initial datum) or the smoothing or non-smoothing effects (depending on the position of $\lambda$ with respect to 1 ).

The plan is as follows. In the next section, we present the numerical method, using only general properties on the discretizations of $\partial_{x}(f(u))$ and $g[u]$ and covering therefore a wide range of possible schemes. The study of this method is done in Section 3, where we prove, thanks to the usual techniques associated with monotone numerical fluxes for conservation laws, the existence of an approximate solution and its convergence toward the (entropy) solution to (1.1). An example of discretization of $g$, satisfying the properties used in the theoretical study of the scheme, is presented in Section 4 along with considerations on the practical implementation; some numerical results are also provided and show the efficiency of the scheme in catching known qualitative properties of the solution to (1.1) (such as the presence of shocks, speed of diffusion, or the asymptotic behavior). A few technical lemmas are gathered in an appendix (Section 5) which closes the article.

\section{Definition of the SCheme AND MAin RESUlt}

Let $\delta t>0$ and $\delta x>0$ be time and space steps. The scheme consists in computing approximate values $u_{i}^{n}$ of the solution to (1.1) on $[n \delta t,(n+1) \delta t[\times[i \delta x,(i+1) \delta x[$ for $n \in \mathbb{N}$ and $i \in \mathbb{Z}$, thanks to the following relations:

$\forall n \geq 0, \forall i \in \mathbb{Z}: \frac{\delta x}{\delta t}\left(u_{i}^{n+1}-u_{i}^{n}\right)+F\left(u_{i}^{n}, u_{i+1}^{n}\right)-F\left(u_{i-1}^{n}, u_{i}^{n}\right)+\delta x g^{\delta x}\left[u^{n+1}\right]_{i}=0$,

$$
\forall i \in \mathbb{Z}: u_{i}^{0}=\frac{1}{\delta x} \int_{i \delta x}^{(i+1) \delta x} u_{0}(x) d x
$$

where $F$ is a numerical flux corresponding to the continuous flux $f$ and $g^{\delta x}$ is a discretization of the non-local term $g$. Notice that the hyperbolic term of the equation is discretized using an explicit method; this imposes a CFL condition on the time and space steps (see (2.4)), but this condition is not very binding and, more importantly, the explicit discretization has the double advantage of avoiding the solving of a non-linear equation at each time step and allowing us to consider as easily more complicated numerical fluxes (see Remark 2.2. higher-order fluxes are not really adapted to an implicit discretization [10]). On the contrary, since the non-local operator is linear and has diffusive properties (similar to the ones of $-\Delta$ ), we use an implicit discretization for $g[u]$ in order not to have to impose, during the proof of a priori estimates on the approximate solution, a more restrictive condition than (2.4) on the time and space steps (see however Section 4.3).

Remark 2.1. Non-uniform time and space steps can as easily be considered but, for the sake of legibility, we only take here uniform $\delta t$ and $\delta x$. 
The numerical fluxes we consider are classical 2-points finite volume monotone fluxes (see [10]):

$$
\begin{gathered}
F: \mathbb{R}^{2} \mapsto \mathbb{R} \text { is Lipschitz continuous on }\left[\inf _{\mathbb{R}} u_{0}, \sup _{\mathbb{R}} u_{0}\right]^{2}, \\
\text { non-decreasing with respect to its first variable, } \\
\text { non-increasing with respect to its second variable, } \\
\quad \text { and } F(a, a)=f(a) \text { for all } a \in\left[\inf _{\mathbb{R}} u_{0}, \sup _{\mathbb{R}} u_{0}\right] .
\end{gathered}
$$

Defining $\operatorname{Lip}_{1, u_{0}}(F)$ and $\operatorname{Lip}_{2, u_{0}}(F)$ as the Lipschitz constants of $F$ with respect to its first and second variable on $\left[\inf _{\mathbb{R}} u_{0}, \sup _{\mathbb{R}} u_{0}\right]^{2}$, it is known that the following CFL condition is required to ensure the stability of explicit schemes involving such monotone fluxes:

$$
\frac{\delta t}{\delta x} \leq \frac{1}{\operatorname{Lip}_{1, u_{0}}(F)+\operatorname{Lip}_{2, u_{0}}(F)} .
$$

Remark 2.2. We write the numerical method and make its theoretical study using basic 2-points fluxes, but nothing prevents us from using higher-order fluxes (computing $f(u)$ at $t=n \delta t$ and $x=i \delta x$ by means of $u_{i-p}^{n}, \ldots, u_{i+q}^{n}$ instead of only $u_{i-1}^{n}$ and $u_{i}^{n}$ ), provided that the scheme they define for $\partial_{t} u+\partial_{x}(f(u))=0$ is stable with respect to the $L^{\infty}$ and $B V$ norms (see Section 3). In particular, in Section 4 , we present numerical results involving 4-points MUSCL fluxes.

For our theoretical study, and as for the numerical fluxes above, the discretization $g^{\delta x}$ of $g$ does not need to have a specific expression but is only required to satisfy a series of assumptions (the curious reader can refer to Section 4.1 for an example of $\left.g^{\delta x}\right)$. The first ones are not surprising since the operator $g$ itself satisfies continuous equivalent formulations of these assumptions (this can easily be seen from (1.2), see 15]):

$$
g^{\delta x}: l^{\infty}(\mathbb{Z}) \mapsto l^{\infty}(\mathbb{Z}) \text { is linear }
$$

$\forall v \in l^{\infty}(\mathbb{Z})$, if $\left(i_{k}\right)_{k \in \mathbb{N}}$ is a sequence in $\mathbb{Z}$ such that $\lim _{k \rightarrow \infty} v_{i_{k}}=\sup _{j \in \mathbb{Z}} v_{j}$,

then $\liminf _{k \rightarrow \infty} g^{\delta x}[v]_{i_{k}} \geq 0$,

$(2.7)$ if $\tau: l^{\infty}(\mathbb{Z}) \mapsto l^{\infty}(\mathbb{Z})$ is the left translation $\tau(v)_{i}=v_{i+1}$, then $\tau g^{\delta x}=g^{\delta x} \tau$.

The next assumption is quite natural in the framework of numerical analysis where, eventually, everything has to be finite in order to be implemented:

$\exists A^{\delta x}>0$ such that, for all $v \in l^{\infty}(\mathbb{Z}), g^{\delta x}[v]_{0}$ only depends on $\left(v_{j}\right)_{|j| \leq A^{\delta x}}$.

The last assumptions impose the consistency of $g^{\delta x}$ as $\delta x \rightarrow 0$ and necessitate introducing a few conventions and notation. If $\delta x$ is a given space step and $v \in$ $l^{\infty}(\mathbb{Z})$, we identify $v$ with the function $v_{\delta x} \in L^{\infty}(\mathbb{R})$ which is piecewise constant equal to $v_{i}$ on $\left[i \delta x,(i+1) \delta x\left[\right.\right.$ for all $i \in \mathbb{Z}$; likewise, $g^{\delta x}[v]$ is either considered as an element of $l^{\infty}(\mathbb{Z})$ or of $L^{\infty}(\mathbb{R})$, depending on the context. If $K$ is a compact subset of $\mathbb{R}, C_{K}^{2}(\mathbb{R})$ is the space of $C^{2}$ functions on $\mathbb{R}$ with support in $K$ (it is endowed with the norm $\left.\|\phi\|_{C_{K}^{2}}=\|\phi\|_{L^{\infty}(\mathbb{R})}+\left\|\phi^{\prime}\right\|_{L^{\infty}(\mathbb{R})}+\left\|\phi^{\prime \prime}\right\|_{L^{\infty}(\mathbb{R})}\right)$ and, for such a function $\phi$, we define $\Phi \in l^{\infty}(\mathbb{Z})$ by $\Phi_{i}=\frac{1}{\delta x} \int_{i \delta x}^{(i+1) \delta x} \phi(x) d x$. $\left(g^{\delta x}\right)^{*}$ is the formal adjoint operator of $g^{\delta x}$ defined by: for all $v \in l^{1}(\mathbb{Z}),\left(g^{\delta x}\right)^{*}[v]_{i}=\sum_{j \in \mathbb{Z}} g^{\delta x}\left[e^{i}\right]_{j} v_{j}$, where $e^{i} \in l^{\infty}(\mathbb{Z})$ is the sequence which has 1 at the $i$-th position and 0 elsewhere 
(note that, since $g$ is selfadjoint, it is probable, but not required, that $g^{\delta x}$ also is selfadjoint). The assumptions regarding the behavior of $g^{\delta x}$ as $\delta x \rightarrow 0$ are:

$\forall K$ compact in $\left.\left.\mathbb{R}, \exists \theta_{K}:\right] 0,1\right] \rightarrow \mathbb{R}^{+}$non-decreasing such that $\lim _{s \rightarrow 0} \theta_{K}(s)=0$, and for all $\phi \in C_{K}^{2}(\mathbb{R})$ and all $\left.\delta x \in\right] 0,1\left[,\left\|\left(g^{\delta x}\right)^{*}[\Phi]-g[\phi]\right\|\left\|_{L^{1}(\mathbb{R})} \leq\right\| \phi \|_{C_{K}^{2}} \theta_{K}(\delta x)\right.$,

$$
\forall r>0, g^{\delta x}=g_{\lambda, r}^{\delta x}+g_{0, r}^{\delta x} \text { where: }
$$

1) $g_{\lambda, r}^{\delta x}$ satisfies (2.5)-(2.8) and (2.9) with $g$ replaced by $g_{\lambda, r}$,

2) $\forall Q$ compact in $\left.\mathbb{R}, \exists \gamma_{r, Q}:\right] 0,1\left[\rightarrow \mathbb{R}^{+}\right.$such that $\lim _{s \rightarrow 0} \gamma_{r, Q}(s)=0$ and, for all $\delta x \in] 0,1\left[\right.$ and all $v \in l^{\infty}(\mathbb{Z}),\left\|g_{0, r}^{\delta x}[v]-g_{0, r}\left[v_{\delta x}\right]\right\|_{L^{1}(Q)} \leq$ $\|v\|_{l^{\infty}(\mathbb{Z})} \gamma_{r, Q}(\delta x)$.

This last assumption is in fact useful only in the case $\lambda \leq 1$, where we have to consider entropy solutions to (1.1) (the entropy formulation of this equation requires cutting $g$ into $g_{\lambda, r}$ and $\left.g_{0, r}\right)$.

Time and space steps $\delta t$ and $\delta x$ being given, in a similar way as above we identify a family $\left(u_{i}^{n}\right)_{n \geq 0}, i \in \mathbb{Z}$ with the function $u_{\delta t, \delta x}:\left[0, \infty\left[\times \mathbb{R} \rightarrow \mathbb{R}\right.\right.$ equal to $u_{i}^{n}$ on $\left[n \delta t,(n+1) \delta t\left[\times\left[i \delta x,(i+1) \delta x\left[\right.\right.\right.\right.$, and $u^{n}: \mathbb{R} \rightarrow \mathbb{R}$ is the function equal to $u_{i}^{n}$ on $[i \delta x,(i+1) \delta x[$. Our main result is the following.

Theorem 2.3 (Existence, uniqueness and convergence of the approximate solution). Assume that (2.3) and (2.5)-(2.9) hold. Then, for all $\delta t>0$ and all $\delta x>0$ satisfying (2.4), there exists a unique bounded solution $u_{\delta t, \delta x}=\left(u_{i}^{n}\right)_{n \geq 0, i \in \mathbb{Z}}$ to (2.1)-(2.2). Moreover, if $\lambda>1$ or if (2.10) holds, then, as $\delta t$ and $\delta x$ tend to 0 (while satisfying (2.4) $), u_{\delta t, \delta x} \rightarrow u$ weakly-* in $L^{\infty}([0, \infty[\times \mathbb{R})$ and strongly in $L_{\mathrm{loc}}^{p}([0, \infty[\times \mathbb{R})$ for all $p<\infty$, where $u$ is the unique entropy solution to (1.1).

Remark 2.4. Since the construction and theoretical study of the scheme do not rely on the precise expression of the non-local term in (1.1), but only on general properties enjoyed by this term and its discretization 1 , Theorem 2.3 can easily be generalized to equations involving, for example, other kinds of Lévy operators (not only the stable operator $g$ ).

\section{Theoretical Study of The SCheme}

3.1. Properties of the approximation $g^{\delta x}$. The assumptions made above on $g^{\delta x}$ allow us to make precise the structure of this discretization of $g$ and to deduce additional properties.

Lemma 3.1. If $g^{\delta x}$ satisfies (2.5)-(2.8), then:

1) $g^{\delta x}$ commutes with the right translation $\tau^{-1}:\left(v_{i}\right)_{i \in \mathbb{Z}} \mapsto\left(v_{i-1}\right)_{i \in \mathbb{Z}}$.

2) If $v \in l^{\infty}(\mathbb{Z})$ and $\left(i_{k}\right)_{k \geq 1}$ are such that $\lim _{k \rightarrow \infty} v_{i_{k}}=\inf _{j \in \mathbb{Z}} v_{j}$, then $\limsup _{k \rightarrow \infty} g^{\delta x}[v]_{i_{k}} \leq 0$.

3) If $v \in l^{\infty}(\mathbb{Z})$ is a constant sequence, then $g^{\delta x}[v]=0$.

4) There exist non-negative real numbers $\left(\mu_{j}^{\delta x}\right)_{j=-A^{\delta x}, \ldots, A^{\delta x}}$ such that

$$
\forall v \in l^{\infty}(\mathbb{Z}), \forall i \in \mathbb{Z}: g^{\delta x}[v]_{i}=-\sum_{|j| \leq A^{\delta x}} \mu_{j}^{\delta x}\left(v_{i+j}-v_{i}\right) .
$$

\footnotetext{
${ }^{1}$ Some of these properties (such as the invariance by translation 2.7) being moreover stated and used only to simplify the presentation.
} 
5) For all $v \in l^{\infty}(\mathbb{Z})$, all $\eta: \mathbb{R} \mapsto \mathbb{R}$ convex functions and all $i \in \mathbb{Z}$, we have

$$
g^{\delta x}[\eta(v)]_{i} \leq \eta^{\prime}\left(v_{i}\right) g^{\delta x}[v]_{i}
$$

(if $\eta$ is not regular, we let $\eta^{\prime}\left(v_{i}\right)$ denote any sub-differential of $\eta$ at $v_{i}$ ).

6) If $v \in l^{\infty}(\mathbb{Z})$ and $\left(v^{m}\right)_{m \geq 1}$ is a bounded sequence in $l^{\infty}(\mathbb{Z})$ such that, for all $i \in \mathbb{Z}, \lim _{m \rightarrow \infty} v_{i}^{m}=v_{i}$, then $\left(g^{\delta x}\left[v^{m}\right]\right)_{m \geq 1}$ is bounded in $l^{\infty}(\mathbb{Z})$ and $\lim _{m \rightarrow \infty} g^{\delta x}\left[v^{m}\right]_{i}=g^{\delta x}[v]_{i}$ for all $i \in \mathbb{Z}$.

7) There exists $C^{\delta x} \geq 0$ such that, for all $v \in l^{\infty}(\mathbb{Z})$ and all $N \geq 1$,

$$
\left|\sum_{i=-N}^{N} g^{\delta x}[v]_{i}\right| \leq C^{\delta x} \sup _{N-A^{\delta x} \leq|i| \leq N+A^{\delta x}}\left|v_{i}\right| .
$$

8) If $w \in l^{1}(\mathbb{Z})$ and $v \in l^{\infty}(\mathbb{Z})$, then $\left(g^{\delta x}\right)^{*}[w] \in l^{1}(\mathbb{Z})$ and

$$
\sum_{i \in \mathbb{Z}} g^{\delta x}[v]_{i} w_{i}=\sum_{i \in \mathbb{Z}} v_{i}\left(g^{\delta x}\right)^{*}[w]_{i}
$$

Remark 3.2. It is shown in 9 that operators acting on spaces of functions on $\mathbb{R}$ and satisfying a reverse maximum principle similar to (2.6) have integral representations, generalizations of (1.2). Formula (3.1) can be seen as a discrete version of this result (see also Section 4.1 to understand the absence, with respect to the continuous case, of a discrete derivative in (3.1) ) and, as in the continuous case, the reverse maximum principle (2.6) truly is the key point to the study of the discretized equation.

Proof of Lemma 3.1. We first notice that Item[1 is evidently true, as a consequence of (2.7) and of the general fact that if an operator commutes with an isomorphism, then it also commutes with its inverse mapping. It is also easy to see that Item 2 is a consequence of 2.6 applied to $-v$ instead of $v$. If $v$ is a constant sequence, then any $i$ satisfies $v_{i}=\sup _{j \in \mathbb{Z}} v_{j}=\inf _{j \in \mathbb{Z}} v_{j}$ and, by (2.6) and Item 2, we must have $g^{\delta x}[v]_{i} \geq 0$ and $g^{\delta x}[v]_{i} \leq 0$, which proves Item 3 .

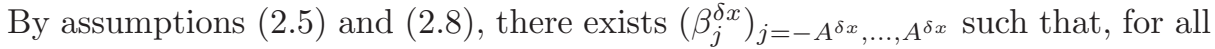
$v \in l^{\infty}(\mathbb{Z})$,

$$
g^{\delta x}[v]_{0}=\sum_{|j| \leq A^{\delta x}} \beta_{j}^{\delta x} v_{j} .
$$

Let $|j| \leq A^{\delta x}, j \neq 0$ and $v \in l^{\infty}(\mathbb{Z})$ be defined by $v_{j}=-1$ and $v_{i}=0$ if $i \neq j$; applying (2.6) with $i_{k} \equiv 0$ (we have $v_{0}=0=\sup _{i \in \mathbb{Z}} v_{i}$ ), we obtain $0 \leq g^{\delta x}[v]_{0}=$ $-\beta_{j}^{\delta x}$, which proves that, for all $j \neq 0, \beta_{j}^{\delta x} \leq 0$. From the invariance by translation (2.7) and Item 1), we also have

$$
g^{\delta x}[v]_{i}=\left(\tau^{i} g^{\delta x}[v]\right)_{0}=g^{\delta x}\left[\tau^{i} v\right]_{0}=\sum_{|j| \leq A^{\delta x}} \beta_{j}^{\delta x}\left(\tau^{i} v\right)_{j}=\sum_{|j| \leq A^{\delta x}} \beta_{j}^{\delta x} v_{i+j}
$$

But Item 3 implies $\sum_{|j| \leq A^{\delta x}} \beta_{j}^{\delta x}=0$ and thus $\beta_{0}^{\delta x}=-\sum_{j \neq 0} \beta_{j}^{\delta x}$, which gives

$$
\begin{aligned}
g^{\delta x}[v]_{i} & =\sum_{j \neq 0} \beta_{j}^{\delta x} v_{i+j}+\beta_{0}^{\delta x} v_{i}=\sum_{j \neq 0} \beta_{j}^{\delta x} v_{i+j}-\left(\sum_{j \neq 0} \beta_{j}^{\delta x}\right) v_{i} \\
& =\sum_{j \neq 0} \beta_{j}^{\delta x}\left(v_{i+j}-v_{i}\right)=\sum_{|j| \leq A^{\delta x}} \beta_{j}^{\delta x}\left(v_{i+j}-v_{i}\right) .
\end{aligned}
$$

Item 4 follows if we define $\mu_{0}^{\delta x}=0$ and, for $j \in\left[-A^{\delta x}, A^{\delta x}\right] \backslash\{0\}, \mu_{j}^{\delta x}=-\beta_{j}^{\delta x}$. 
If $\eta$ is convex, then $\eta\left(v_{i+j}\right)-\eta\left(v_{i}\right) \geq \eta^{\prime}\left(v_{i}\right)\left(v_{i+j}-v_{i}\right)$ and Item 5 is thus a corollary of Item 4. Item 6 is also an immediate consequence of Formula (3.1) and, to prove Item 7 , we simply write

$$
\begin{aligned}
\sum_{i=-N}^{N} g^{\delta x}[v]_{i}= & \sum_{|j| \leq A^{\delta x}} \beta_{j}^{\delta x}\left(\sum_{i=-N}^{N} v_{i+j}-\sum_{i=-N}^{N} v_{i}\right) \\
= & \sum_{0 \leq j \leq A^{\delta x}} \beta_{j}^{\delta x}\left(\sum_{i=N+1}^{N+j} v_{i}-\sum_{i=-N}^{-N+j-1} v_{i}\right) \\
& +\sum_{-A^{\delta x} \leq j<0} \beta_{j}^{\delta x}\left(\sum_{i=-N+j}^{-N-1} v_{i}-\sum_{i=N+j+1}^{N} v_{i}\right)
\end{aligned}
$$

and thus

$$
\left|\sum_{i=-N}^{N} g^{\delta x}[v]_{i}\right| \leq 4 \sum_{|j| \leq A^{\delta x}} j \beta_{j}^{\delta x} \times \sup _{N-A^{\delta x} \leq|i| \leq N+A^{\delta x}}\left|v_{i}\right| .
$$

It remains to prove Item 8. First, by (3.1), it is easy to see that $\left(g^{\delta x}\right)^{*}$ satisfies the same formula with $\mu_{-j}^{\delta x}$ instead of $\mu_{j}^{\delta x}$; hence, if $w \in l^{1}(\mathbb{Z})$, then $\left(g^{\delta x}\right)^{*}[w]$ is also in $l^{1}(\mathbb{Z})$. By definition of $\left(g^{\delta x}\right)^{*}$ and linearity of $g^{\delta x},(3.3)$ is true if $v$ has only a finite number of non-zero terms; since we can approximate, term by term, any $v \in l^{\infty}(\mathbb{Z})$ by such sequences which stay bounded in $l^{\infty}(\mathbb{Z}),(3.3)$ for a general $v$ follows from Item 6 .

3.2. Existence and uniqueness of an approximate solution. We prove in this section that there exists a unique solution to (2.1)-(2.2), and we establish a first series of properties of this solution.

Lemma 3.3. Under assumptions (2.5)- for all $\alpha \geq 0$ and all $h \in l^{\infty}(\mathbb{Z})$, there exists a unique solution $v \in l^{\infty}(\mathbb{Z})$ to

$$
\forall i \in \mathbb{Z}: v_{i}+\alpha g^{\delta x}[v]_{i}=h_{i} .
$$

Moreover, we have

$$
\inf _{i \in Z} h_{i} \leq \inf _{i \in \mathbb{Z}} v_{i} \leq \sup _{i \in \mathbb{Z}} v_{i} \leq \sup _{i \in \mathbb{Z}} h_{i}
$$

and

$$
\sum_{i \in \mathbb{Z}}\left|v_{i}\right| \leq \sum_{i \in \mathbb{Z}}\left|h_{i}\right|
$$

Proof of Lemma 3.3. Let us first prove (3.5) and the uniqueness of the solution. Let $\left(i_{k}\right)_{k \geq 1}$ be a sequence in $\mathbb{Z}$ such that $\lim _{k \rightarrow \infty} v_{i_{k}}=\sup _{i \in \mathbb{Z}} v_{i}$; then applying (3.4) to $i=i_{k}$ and passing to the inferior limit as $k \rightarrow \infty$ thanks to (2.6) we find $\sup _{i \in \mathbb{Z}} v_{i} \leq \sup _{i \in Z} h_{i}$. Doing the same along a subsequence which converges to $\inf _{i \in \mathbb{Z}} v_{i}$ (see item 2 in Lemma 3.1), we obtain $\inf _{i \in \mathbb{Z}} v_{i} \geq \inf _{i \in Z} h_{i}$ and (3.5) is proved. These inequalities show that if $h=0$, then $v=0$. System (3.4) being linear, this proves the uniqueness of its solution.

To prove the existence of a solution we consider, for $m \geq 1$, the approximate problem

$$
\forall i \in \mathbb{Z}: v_{i}^{m}+\alpha g^{\delta x}\left[v^{m}\right]_{i} \mathbf{1}_{[-m, m]}(i)=h_{i},
$$


where $\mathbf{1}_{[-m, m]}(i)=1$ if $|i| \leq m$ and $\mathbf{1}_{[-m, m]}(i)=0$ otherwise. Using the same argument as before, we notice that any solution to (3.7) also satisfies (3.5) and, in particular, that this problem has at most one solution. Since (3.7) clearly defines $v_{i}^{m}$ (equal to $h_{i}$ ) if $|i|>m$, solving this system comes down to solving a finitedimensional square linear system (of size $2 m+1$ ); the uniqueness of the solution therefore ensures its existence.

Since $\left(v^{m}\right)_{m>1}$ is bounded in $l^{\infty}(\mathbb{Z})$ (it satisfies 3.5$)$ ), we can assume up to a subsequence that, for all $i \in \mathbb{Z},\left(v_{i}^{m}\right)_{m \geq 1}$ converges to some $v_{i}$ as $m \rightarrow \infty$. We can then pass to the limit $m \rightarrow \infty$ in 3.7 thanks to Item 6 in Lemma 3.1] to see that $\left(v_{i}\right)_{i \in \mathbb{Z}} \in l^{\infty}(\mathbb{Z})$ thus defined satisfies (3.4).

We conclude by proving (3.6), assuming that $h \in l^{1}(\mathbb{Z})$ (otherwise nothing needs to be proved). Multiplying (3.4) by $\operatorname{sgn}\left(v_{i}\right)=\eta^{\prime}\left(v_{i}\right)$ for $\eta=|\cdot|$ and using (3.2), we have $\left|v_{i}\right|+\alpha g^{\delta x}[|v|]_{i} \leq\left|h_{i}\right|$. Summing on $i=-N, \ldots, N$, we deduce from Item 7 in Lemma 3.1 that

$\sum_{i=-N}^{N}\left|v_{i}\right| \leq \sum_{i=-N}^{N}\left|h_{i}\right|+\alpha C^{\delta x} \sup _{N-A^{\delta x} \leq|i| \leq N+A^{\delta x}}\left|v_{i}\right| \leq \sum_{i \in \mathbb{Z}}\left|h_{i}\right|+\alpha C^{\delta x}\|v\|_{l^{\infty}(\mathbb{Z})}<+\infty$.

Hence $v \in l^{1}(\mathbb{Z})$ and $\lim _{|i| \rightarrow \infty} v_{i}=0$. We infer that $\lim _{N \rightarrow \infty} \sup _{N-A^{\delta x} \leq|i| \leq N+A^{\delta x}}\left|v_{i}\right|$ $=0$ and, letting $N \rightarrow \infty$ in the first inequality of (3.8), this concludes the proof of (3.6).

We can now prove the existence and uniqueness of the solution to the scheme.

Corollary 3.4 (Existence and uniqueness of an approximate solution). Let $\delta t>0$ and $\delta x>0$. Under assumptions (2.3)-(2.8), there exists a unique bounded solution $\left(u_{i}^{n}\right)_{n \geq 0, i \in \mathbb{Z}}$ to (2.1)-(2.2). Moreover, it satisfies, for all $n \geq 1$,

$$
\inf _{\mathbb{R}} u_{0} \leq \inf _{i \in \mathbb{Z}} u_{i}^{n} \leq \sup _{i \in \mathbb{Z}} u_{i}^{n} \leq \sup _{\mathbb{R}} u_{0}
$$

Proof of Corollary 3.4. As is usual for schemes involving monotone fluxes, we rewrite (2.2) in the following way:

$$
\begin{aligned}
u_{i}^{n+1}+\delta t g^{\delta x}\left[u^{n+1}\right]_{i}= & u_{i}^{n}-\frac{\delta t}{\delta x}\left(F\left(u_{i}^{n}, u_{i+1}^{n}\right)-F\left(u_{i}^{n}, u_{i}^{n}\right)\right) \\
& +\frac{\delta t}{\delta x}\left(F\left(u_{i-1}^{n}, u_{i}^{n}\right)-F\left(u_{i}^{n}, u_{i}^{n}\right)\right) \\
= & u_{i}^{n}-\frac{\delta t}{\delta x} \frac{F\left(u_{i}^{n}, u_{i+1}^{n}\right)-F\left(u_{i}^{n}, u_{i}^{n}\right)}{u_{i+1}^{n}-u_{i}^{n}}\left(u_{i+1}^{n}-u_{i}^{n}\right) \\
& +\frac{\delta t}{\delta x} \frac{F\left(u_{i-1}^{n}, u_{i}^{n}\right)-F\left(u_{i}^{n}, u_{i}^{n}\right)}{u_{i-1}^{n}-u_{i}^{n}}\left(u_{i-1}^{n}-u_{i}^{n}\right) .
\end{aligned}
$$

Let us define

$$
a_{i}^{n}=-\frac{\delta t}{\delta x} \frac{F\left(u_{i}^{n}, u_{i+1}^{n}\right)-F\left(u_{i}^{n}, u_{i}^{n}\right)}{u_{i+1}^{n}-u_{i}^{n}} \quad \text { and } \quad b_{i}^{n}=\frac{\delta t}{\delta x} \frac{F\left(u_{i-1}^{n}, u_{i}^{n}\right)-F\left(u_{i}^{n}, u_{i}^{n}\right)}{u_{i-1}^{n}-u_{i}^{n}}
$$

(if $u_{i+1}^{n}=u_{i}^{n}$ or $u_{i-1}^{n}=u_{i}^{n}$, we let the corresponding coefficient be equal to zero). The scheme is thus equivalent to

$$
u_{i}^{n+1}+\delta t g^{\delta x}\left[u^{n+1}\right]_{i}=u_{i}^{n}+a_{i}^{n}\left(u_{i+1}^{n}-u_{i}^{n}\right)+b_{i}^{n}\left(u_{i-1}^{n}-u_{i}^{n}\right),
$$


which comes down to asking that $u^{n+1}$ be the solution to 3.4 with $\alpha=\delta t$ and $h_{i}=\left(1-a_{i}^{n}-b_{i}^{n}\right) u_{i}^{n}+a_{i}^{n} u_{i+1}^{n}+b_{i}^{n} u_{i-1}^{n}$. But, under (2.3) and (2.4), if $u^{n}$ satisfies (3.9), then $a_{i}^{n} \geq 0, b_{i}^{n} \geq 0$ and $a_{i}^{n}+b_{i}^{n} \leq 1$; this means that $h_{i}$ is a convex combination of $\left(u_{j}^{n}\right)_{j \in \mathbb{Z}}$ and thus that $\inf _{i \in \mathbb{Z}} u_{i}^{n} \leq \inf _{i \in \mathbb{Z}} h_{i} \leq \sup _{i \in \mathbb{Z}} h_{i} \leq \sup _{i \in \mathbb{Z}} u_{i}^{n}$. Hence, reasoning by induction on $n$ from (2.1), Lemma 3.3 ensures the existence and uniqueness of a bounded solution to (2.1) (2.2), which satisfies moreover (3.9).

\subsection{Compactness estimates.}

Proposition 3.5 ( $B V$ estimates). Let $\delta t>0$ and $\delta x>0$ and assume (2.3)-(2.8). If $\left(u_{i}^{n}\right)_{n \geq 0, i \in \mathbb{Z}}$ is the solution to (2.1)-(2.2), then, for all $n \geq 1$,

$$
\sum_{i \in \mathbb{Z}}\left|u_{i+1}^{n}-u_{i}^{n}\right| \leq\left|u_{0}\right|_{B V(\mathbb{R})} .
$$

Proof of Proposition 3.5. Subtracting (3.10) for $i+1$ and for $i$ and since $g^{\delta x}$ commutes with the translation $\tau$, we obtain, with $v_{i}^{n}=u_{i+1}^{n}-u_{i}^{n}$,

$$
v_{i}^{n+1}+\delta t g^{\delta x}\left[v^{n+1}\right]_{i}=\left(1-a_{i}^{n}-b_{i+1}^{n}\right) v_{i}^{n}+a_{i+1}^{n} v_{i+1}^{n}+b_{i}^{n} v_{i-1}^{n} .
$$

Hence $v^{n+1}$ is the solution to (3.4) with $\alpha=\delta t$ and $h_{i}=\left(1-a_{i}^{n}-b_{i+1}^{n}\right) v_{i}^{n}+$ $a_{i+1}^{n} v_{i+1}^{n}+b_{i}^{n} v_{i-1}^{n}$ and we deduce from (3.6) that

$$
\sum_{i \in \mathbb{Z}}\left|v_{i}^{n+1}\right| \leq \sum_{i \in \mathbb{Z}}\left|1-a_{i}^{n}-b_{i+1}^{n}\right|\left|v_{i}^{n}\right|+\sum_{i \in \mathbb{Z}}\left|a_{i+1}^{n}\right|\left|v_{i+1}^{n}\right|+\sum_{i \in \mathbb{Z}}\left|b_{i}^{n}\right|\left|v_{i-1}^{n}\right| .
$$

But the CFL confition (2.4) and Estimate (3.9) ensure that $1-a_{i}^{n}-b_{i+1}^{n} \geq 0$, $a_{i+1}^{n} \geq 0$ and $b_{i}^{n} \geq 0$ and we therefore find, by reindexing the last two sums,

$$
\sum_{i \in \mathbb{Z}}\left|u_{i+1}^{n+1}-u_{i}^{n+1}\right| \leq \sum_{i \in \mathbb{Z}}\left|u_{i+1}^{n}-u_{i}^{n}\right| .
$$

This estimate allows us to conclude the proof by induction on $n$ (because (3.11) is true for $n=0$ from the definition of $u^{0}$ in (2.1); see [10]).

Proposition 3.6 (Time estimates). Let $\delta t>0$ and $\delta x \in] 0,1[$. Assume that (2.3) (2.9) hold and let $u_{\delta t, \delta x}=\left(u_{i}^{n}\right)_{n \geq 0, i \in \mathbb{Z}}$ be the solution to (2.1)-(2.2). Define $\widetilde{u}_{\delta t, \delta x}$ as the affine by parts time interpolate of $\left(u_{i}^{n}\right)_{n \geq 0, i \in \mathbb{Z}}$ :

$\forall t \in[n \delta t,(n+1) \delta t], \forall x \in \mathbb{R}: \widetilde{u}_{\delta t, \delta x}(t, x)=\frac{t-n \delta t}{\delta t} u^{n+1}(x)+\frac{(n+1) \delta t-t}{\delta t} u^{n}(x)$.

Then for all compact subsets $K$ of $\mathbb{R}$, there exists $M_{K} \geq 0$ not depending on $\delta t$ or $\delta x$ such that

$$
\left.|| \partial_{t} \widetilde{u}_{\delta t, \delta x}\right|_{L^{\infty}(] 0, \infty\left[;\left(C_{K}^{2}(\mathbb{R})\right)^{\prime}\right)} \leq M_{K}
$$

Remark 3.7. If $\lambda<1$, then (1.2) shows that $g[\phi] \in L_{\text {loc }}^{1}(\mathbb{R})$ as soon as $\phi \in L^{\infty}(\mathbb{R}) \cap$ $B V(\mathbb{R})$. Hence, since Proposition 3.5 gives space $B V$ estimates on $u_{\delta t, \delta x}$, the choice of a proper approximation $g^{\delta x}$ and the scheme (2.2) could allow us to deduce, in the case $\lambda<1$, time- $B V$ local estimates on $u_{\delta t, \delta x}$ (stronger estimates than (3.12)). 
Proof of Proposition 3.6. We have $\partial_{t} \widetilde{u}=\frac{u^{n+1}-u^{n}}{\delta t}$ on $[n \delta t,(n+1) \delta t] \times \mathbb{R}$. Let $K$ be a compact subset in $\mathbb{R}$ and $\phi \in C_{K}^{2}(\mathbb{R})$; define $\Phi \in l^{\infty}(\mathbb{Z})$ by $\Phi_{i}=\frac{1}{\delta x} \int_{i \delta x}^{(i+1) \delta x} \phi(x) d x$. From (2.2) we deduce, for $t \in[n \delta t,(n+1) \delta t]$,

$$
\begin{aligned}
\int_{\mathbb{R}} \partial_{t} \widetilde{u}_{\delta t, \delta x}(t, x) \phi(x) d x & =\sum_{i \in \mathbb{Z}} \frac{\delta x}{\delta t}\left(u_{i}^{n+1}-u_{i}^{n}\right) \Phi_{i} \\
(3.13) & =\sum_{i \in \mathbb{Z}}\left(F\left(u_{i-1}^{n}, u_{i}^{n}\right)-F\left(u_{i}^{n}, u_{i+1}^{n}\right)\right) \Phi_{i}-\sum_{i \in \mathbb{Z}} \delta x g^{\delta x}\left[u^{n+1}\right]_{i} \Phi_{i} .
\end{aligned}
$$

Using (2.3), we have $\left|F\left(u_{i-1}^{n}, u_{i}^{n}\right)-F\left(u_{i}^{n}, u_{i+1}^{n}\right)\right| \leq C_{1}\left(\left|u_{i+1}^{n}-u_{i}^{n}\right|+\left|u_{i}^{n}-u_{i-1}^{n}\right|\right)$ with $C_{\text {日 }}$ not depending on $\delta t, \delta x, n$ or $i$, and (3.11) therefore gives

$$
\left|\sum_{i \in \mathbb{Z}}\left(F\left(u_{i-1}^{n}, u_{i}^{n}\right)-F\left(u_{i}^{n}, u_{i+1}^{n}\right)\right) \Phi_{i}\right| \leq 2 C_{\mathbb{1}}\left|u_{0}\right|_{B V(\mathbb{R})}|| \phi \|_{L^{\infty}(\mathbb{R})} .
$$

Formula (1.2) clearly shows that $g$ is continuous $W^{2,1}(\mathbb{R}) \rightarrow L^{1}(\mathbb{R})$ and thus, since $C_{K}^{2}(\mathbb{R})$ is continuously embedded in $W^{2,1}(\mathbb{R})$, there exists $E_{K}$ not depending on $\phi$ such that $\left.\|g[\phi]\|\right|_{L^{1}(\mathbb{R})} \leq E_{K}\|\phi\|_{C_{K}^{2}}$; using Item 8 in Lemma 3.1, (2.9) and (3.9), we deduce

$$
\begin{aligned}
\left|\sum_{i \in \mathbb{Z}} \delta x g^{\delta x}\left[u^{n+1}\right]_{i} \Phi_{i}\right| & =\left|\sum_{i \in \mathbb{Z}} \delta x u_{i}^{n+1}\left(g^{\delta x}\right)^{*}[\Phi]_{i}\right| \\
& \leq\left.\left\|u_{0}\right\|_{L^{\infty}(\mathbb{R})}\left\|\left(g^{\delta x}\right)^{*}[\Phi]\right\|\right|_{L^{1}(\mathbb{R})} \\
& \leq\left\|u_{0}\right\|_{L^{\infty}(\mathbb{R})}\left(\theta_{K}(1)+E_{K}\right)\|\phi\|_{C_{K}^{2}} .
\end{aligned}
$$

The proof is concluded by plugging (3.14) and (3.15) into (3.13).

Corollary 3.8 (Compactness of the approximate solution). Assume that (2.3) and (2.5) -(2.9) hold. Then as $\delta t>0$ and $\delta x>0$ tend to 0 while satisfying (2.4), up to a subsequence the solution $u_{\delta t, \delta x}$ to (2.1) -(2.2) converges in $L_{\mathrm{loc}}^{1}([0, \infty[\times \mathbb{R})$.

Proof of Corollary 3.8. Let $\delta t>0$ and $\delta x \in] 0,1\left[\right.$ satisfy (2.4) and define $\widetilde{u}_{\delta t, \delta x}$ as the affine interpolate of $\left(u_{i}^{n}\right)_{n \geq 0,, i \in \mathbb{Z}}$ as in Proposition 3.6. Estimate (3.11) shows that, for all $n \geq 0,\left|u^{n}\right|_{B V(\mathbb{R})} \leq\left|u_{0}\right|_{B V(\mathbb{R})}$; since, for all $t>0, \widetilde{u}_{\delta t, \delta x}(t, \cdot)$ is a convex combination of $u^{n}$ and $u^{n+1}$ (for some $n \geq 0$ ), we deduce that $\left|\widetilde{u}_{\delta t, \delta x}(t, \cdot)\right|_{B V(\mathbb{R})} \leq$ $\left|u_{0}\right|_{B V(\mathbb{R})}$ and, by (3.9), that $\left\|\widetilde{u}_{\delta t, \delta x}\right\|_{L^{\infty}(10, \infty[\times \mathbb{R})} \leq\left\|u_{0}\right\|_{L^{\infty}(\mathbb{R})}$.

For all compact $K \subset \mathbb{R}$, the set $S=\left\{\widetilde{u}_{\delta t, \delta x} \mid \delta t>0\right.$ and $\delta x>0$ satisfy (2.4) $\}$ is therefore bounded in $L^{\infty}\left(\left[0, \infty\left[; L^{\infty}(K)\right)\right.\right.$ and, by Proposition 3.6. the time derivatives of the functions in this set are bounded in $L^{\infty}\left(\left[0, \infty\left[;\left(C_{K}^{2}(\mathbb{R})\right)^{\prime}\right)\right.\right.$. Since $L^{\infty}(K)$ is compactly embedded in $\left(C_{K}^{2}(\mathbb{R})\right)^{\prime}$ (because $C_{K}^{2}(\mathbb{R})$ is compactly and densely embedded in $\left.L^{1}(K)\right)$, we deduce that $S$ is bounded in $W^{1, \infty}\left(\left[0, \infty\left[;\left(C_{K}^{2}(\mathbb{R})\right)^{\prime}\right)\right.\right.$ and, by Aubin-Simon's compactness theorem (see [4, 21]), that $S$ is relatively compact in $L_{\text {loc }}^{1}\left(\left[0, \infty\left[;\left(C_{K}^{2}(\mathbb{R})\right)^{\prime}\right)\right.\right.$.

For all $t \geq 0$, denoting by $n$ the integer such that $t \in[n \delta t,(n+1) \delta t[$, we have $u_{\delta t, \delta x}(t, \cdot)=\widetilde{u}_{\delta t, \delta x}(n \delta t, \cdot)$, and the bound in $W^{1, \infty}\left(\left[0, \infty\left[;\left(C_{K}^{2}(\mathbb{R})\right)^{\prime}\right)\right.\right.$ thus shows that $\left\|u_{\delta t, \delta x}(t, \cdot)-\widetilde{u}_{\delta t, \delta x}(t, \cdot)\right\|_{\left(C_{K}^{2}(\mathbb{R})\right)^{\prime}} \leq C_{2} \delta t$ with $C_{2}$ not depending on $\delta t$ or $\delta x$. The compactness of $S$ in $L_{\text {loc }}^{1}\left(\left[0, \infty\left[;\left(C_{K}^{2}(\mathbb{R})\right)^{\prime}\right)\right.\right.$ therefore shows that, as $\delta t \rightarrow 0$, $u_{\delta t, \delta x}$ is also relatively compact in this space. 
By (3.11), for all $t \geq 0$ we have $\left|u_{\delta t, \delta x}(t, \cdot)\right|_{B V(\mathbb{R})} \leq\left|u_{0}\right|_{B V(\mathbb{R})}$, which implies, by a classical result on $B V$ functions, for all $\xi \in \mathbb{R}$,

$$
\left\|u_{\delta t, \delta x}(t, \cdot+\xi)-u_{\delta t, \delta x}(t, \cdot)\right\|_{L^{1}(\mathbb{R})} \leq\left|u_{0}\right|_{B V(\mathbb{R})}|\xi| .
$$

Associated with the relative compactness, as $\delta t \rightarrow 0$, of $u_{\delta t, \delta x}$ in $L_{\mathrm{loc}}^{1}([0, \infty[$; $\left.\left(C_{K}^{2}(\mathbb{R})\right)^{\prime}\right)$ for all $K \subset \mathbb{R}$ compact, this estimate makes it possible to apply Lemma 7.5 in [1] (or more precisely the technique of proof of this lemma) to conclude that this relative compactness also holds in $L_{\mathrm{loc}}^{1}\left(\left[0, \infty\left[; L_{\mathrm{loc}}^{1}(\mathbb{R})\right)\right.\right.$.

Remark 3.9. If $u_{0}$ does not belong to $B V(\mathbb{R})$, then it is not possible in general to directly prove strong space $B V$ estimates, and thus strong compactness, for $u_{\delta t, \delta x}$. In this situation, one has to invoke the convergence of $u_{\delta t, \delta x}$ in the non-linear $L^{\infty}$ weak-* sense (i.e. in the sense of Young measures) to prove that the limit of $u_{\delta t, \delta x}$ is an entropy process solution to (1.1) (this is done thanks to some space weak $B V$ estimates on $\left.u_{\delta t, \delta x}\right)$ and to check, following [1, that this entropy process solution is unique (see the general method for pure scalar conservation laws in [10]).

3.4. Convergence. We can now prove the convergence of the solution of (2.1) (2.2) toward the solution of (1.1), as $\delta t$ and $\delta x$ tend to 0 while satisfying (2.4). By Corollary 3.8 and since $u_{\delta t, \delta x}$ is bounded in $L^{\infty}([0, \infty[\times \mathbb{R})$, up to a subsequence we can assume that it converges toward some $u$ weak- $*$ in $L^{\infty}([0, \infty[\times \mathbb{R})$ and strongly in $L_{\text {loc }}^{p}\left(\left[0, \infty[\times \mathbb{R})\right.\right.$ for all $p<\infty$. We now show that any such limit $u$ of $u_{\delta t, \delta x}$ is the unique (entropy) solution to (1.1), which implies that the whole family $u_{\delta t, \delta x}$ converges to this solution and concludes the proof of Theorem 2.3 .

Let $\phi \in C_{c}^{2}\left(\left[0, \infty[\times \mathbb{R})\right.\right.$ and define $\Phi_{i}^{n}=\frac{1}{\delta x} \int_{i \delta x}^{(i+1) \delta x} \phi(n \delta t, x) d x$. Multiplying (2.2) by $\delta t \Phi_{i}^{n}$ and summing on $n$ and $i$ (all these sums are finite since $\Phi_{i}^{n}$ is equal to zero for $n$ or $|i|$ large), we obtain $T_{1}+T_{2}+T_{3}=0$, where

$$
\begin{gathered}
T_{\text {百 }}=\sum_{n \geq 0} \sum_{i \in \mathbb{Z}} \delta x\left(u_{i}^{n+1}-u_{i}^{n}\right) \Phi_{i}^{n}, \\
T_{\text {目 }}=\sum_{n \geq 0} \delta t \sum_{i \in \mathbb{Z}}\left(F\left(u_{i}^{n}, u_{i+1}^{n}\right)-F\left(u_{i-1}^{n}, u_{i}^{n}\right)\right) \Phi_{i}^{n}
\end{gathered}
$$

and

$$
T_{3}=\sum_{n \geq 0} \delta t \sum_{i \in \mathbb{Z}} \delta x g^{\delta x}\left[u^{n+1}\right]_{i} \Phi_{i}^{n} .
$$

Let us study the limit of each of these terms. We have

$$
\begin{aligned}
T_{\text {回 }} & =\sum_{n \geq 1} \delta t \sum_{i \in \mathbb{Z}} \delta x u_{i}^{n} \frac{\Phi_{i}^{n-1}-\Phi_{i}^{n}}{\delta t}-\sum_{i \in \mathbb{Z}} \delta x u_{i}^{0} \Phi_{i}^{0} \\
& =\int_{0}^{\infty} \int_{\mathbb{R}} u_{\delta t, \delta x}(t, x) \Psi_{\delta t, \delta x}(t, x) d t d x-\int_{\mathbb{R}} u_{0}(x) \Phi^{0}(x) d x,
\end{aligned}
$$

where $\Psi_{\delta t, \delta x}$ is equal to 0 on $\left[0, \delta t\left[\times \mathbb{R}\right.\right.$ and to $\frac{\Phi_{i}^{n-1}-\Phi_{i}^{n}}{\delta t}$ on $[n \delta t,(n+1) \delta t[\times[i \delta x,(i+$ 1) $\delta x$ [ for all $n \geq 1$ and all $i \in \mathbb{Z}$. By regularity of $\phi$, as $\delta t$ and $\delta x$ tend to $0, \Psi_{\delta t, \delta x}$ and $\Phi^{0}$ converge respectively to $-\partial_{t} \phi$ in $L^{1}\left(\left[0, \infty[\times \mathbb{R})\right.\right.$ and to $\phi(0, \cdot)$ in $L^{1}(\mathbb{R})$. The weak-* convergence of $u_{\delta t, \delta x}$ then shows that

$$
\text { 畐 } \rightarrow-\int_{0}^{\infty} \int_{\mathbb{R}} u(t, x) \partial_{t} \phi(t, x) d t d x-\int_{\mathbb{R}} u_{0}(x) \phi(0, x) d x .
$$


To handle T国 we write, thanks to (2.3),

$$
\begin{aligned}
T_{\text {圂 }}= & \sum_{n \geq 0} \delta t \sum_{i \in \mathbb{Z}} \delta x F\left(u_{i}^{n}, u_{i+1}^{n}\right) \frac{\Phi_{i}^{n}-\Phi_{i+1}^{n}}{\delta x} \\
= & \sum_{n \geq 0} \delta t \sum_{i \in \mathbb{Z}} \delta x f\left(u_{i}^{n}\right) \frac{\Phi_{i}^{n}-\Phi_{i+1}^{n}}{\delta x} \\
& +\sum_{n \geq 0} \delta t \sum_{i \in \mathbb{Z}} \delta x\left(F\left(u_{i}^{n}, u_{i+1}^{n}\right)-f\left(u_{i}^{n}\right)\right) \frac{\Phi_{i}^{n}-\Phi_{i+1}^{n}}{\delta x} \\
= & \int_{0}^{\infty} \int_{\mathbb{R}} f\left(u_{\delta t, \delta x}(t, x)\right) \Theta_{\delta t, \delta x}(t, x) d t d x \\
& +\sum_{n \geq 0} \delta t \sum_{i \in \mathbb{Z}} \delta x\left(F\left(u_{i}^{n}, u_{i+1}^{n}\right)-F\left(u_{i}^{n}, u_{i}^{n}\right)\right) \frac{\Phi_{i}^{n}-\Phi_{i+1}^{n}}{\delta x},
\end{aligned}
$$

where $\Theta_{\delta t, \delta x}=\frac{\Phi_{i}^{n}-\Phi_{i+1}^{n}}{\delta x}$ on $[n \delta t,(n+1) \delta t[\times[i \delta x,(i+1) \delta x[$ for all $n \geq 0$ and all $i \in \mathbb{Z} ;$ as $\delta t$ and $\delta x$ tend to 0 , this function converges to $-\partial_{x} \phi$ in $L^{1}([0, \infty[\times \mathbb{R})$ by regularity of $\phi$. Moreover, by local Lipschitz continuity of $f$, uniform bound on $u_{\delta t, \delta x}$ and convergence of this function toward $u$ in $L_{\text {loc }}^{1}\left(\left[0, \infty[\times \mathbb{R}), f\left(u_{\delta t, \delta x}\right) \rightarrow\right.\right.$ $f(u)$ in $L_{\text {loc }}^{1}\left(\left[0, \infty[\times \mathbb{R})\right.\right.$ while staying bounded in $L^{\infty}([0, \infty[\times \mathbb{R})$; the convergence of $f\left(u_{\delta t, \delta x}\right)$ thus also holds in $L^{\infty}([0, \infty[\times \mathbb{R})$ weak-* and we therefore see that the first term on the right-hand side of (3.17) tends to $-\int_{0}^{\infty} \int_{\mathbb{R}} f(u(t, x)) \partial_{x} \phi(t, x) d t d x$. Regarding the second term, we invoke (3.11) and the regularity of $\phi$ to write

$$
\begin{gathered}
\left|\sum_{n \geq 0} \delta t \sum_{i \in \mathbb{Z}} \delta x\left(F\left(u_{i}^{n}, u_{i+1}^{n}\right)-F\left(u_{i}^{n}, u_{i}^{n}\right)\right) \frac{\Phi_{i}^{n}-\Phi_{i+1}^{n}}{\delta x}\right| \\
\leq \operatorname{Lip}_{2, u_{0}}(F) C_{3} \sum_{0 \leq n<T / \delta t} \delta t \sum_{i \in \mathbb{Z}} \delta x\left|u_{i+1}^{n}-u_{i}^{n}\right| \\
\leq \operatorname{Lip}_{2, u_{0}}(F) C_{3} T\left|u_{0}\right|_{B V(\mathbb{R})} \delta x,
\end{gathered}
$$

where $C_{3}$ only depends on $\phi$, and $T$ is such that $\operatorname{supp}(\phi) \subset[0, T] \times \mathbb{R}$ (so that $\Phi_{i}^{n}=0$ if $n \geq T / \delta t$ ). This last right-hand side tends to 0 with $\delta x$ and we conclude that

$$
\text { T国 } \rightarrow-\int_{0}^{\infty} \int_{\mathbb{R}} f(u(t, x)) \partial_{x} \phi(t, x) d t d x .
$$

The convergence of $T_{3}$ is pretty straightforward from Item 8 in Lemma 3.1 and assumption (2.9): we have

$$
T_{[3}=\sum_{n \geq 0} \delta t \sum_{i \in \mathbb{Z}} \delta x u_{i}^{n+1}\left(g^{\delta x}\right)^{*}\left[\Phi^{n}\right]_{i}=\int_{0}^{T} \int_{\mathbb{R}} u_{\delta t, \delta x}(t+\delta t, x) \Omega_{\delta t, \delta x}(t, x) d t d x
$$

where $\Omega_{\delta t, \delta x}=\left(g^{\delta x}\right)^{*}\left[\Phi^{n}\right]_{i}$ on $[n \delta t,(n+1) \delta t[\times[i \delta x,(i+1) \delta x[$ for all $n \geq 0$ and all $i \in \mathbb{Z}$ and $T$ is as before. Let $\phi_{\delta t}:[0, \infty[\times \mathbb{R} \rightarrow \mathbb{R}$ be the function equal to $\phi(n \delta t, \cdot)$ on $[n \delta t,(n+1) \delta t[\times \mathbb{R}$ for all $n \geq 0$. From (2.9) we have, for all $t \geq 0,\left\|\Omega_{\delta t, \delta x}(t, \cdot)-g\left[\phi_{\delta t}(t, \cdot)\right]\right\|_{L^{1}(\mathbb{R})} \leq\|\phi\|_{L^{\infty}\left(\left[0, \infty\left[; C_{K}^{2}(\mathbb{R})\right)\right.\right.} \theta_{K}(\delta x)$, where $K$ is a compact set such that $\operatorname{supp}(\phi) \subset[0, \infty[\times K$. As $\delta t \rightarrow 0$, the regularity of $\phi$ ensures that $\phi_{\delta t} \rightarrow \phi$ in $L^{\infty}\left([0, T] ; C_{K}^{2}(\mathbb{R})\right)$, and thus in $L^{\infty}\left([0, T] ; W^{2,1}(\mathbb{R})\right)$; since $g: W^{2,1}(\mathbb{R}) \rightarrow L^{1}(\mathbb{R})$ is linear continuous (see Formula $(1.2)$ ), this shows that 
$g\left[\phi_{\delta t}\right] \rightarrow g[\phi]$ in $L^{\infty}\left([0, T] ; L^{1}(\mathbb{R})\right)$. We deduce that, as $\delta t$ and $\delta x$ tend to 0 , $\Omega_{\delta t, \delta x} \rightarrow g[\phi]$ in $L^{\infty}\left([0, T] ; L^{1}(\mathbb{R})\right) \hookrightarrow L^{1}([0, T] \times \mathbb{R})$. Passing to the limit in (3.19) by weak-* convergence in $L^{\infty}\left(\left[0, \infty[\times \mathbb{R})\right.\right.$ of $u_{\delta t, \delta x}$, we find

$$
T_{[3} \rightarrow \int_{0}^{T} \int_{\mathbb{R}} u(t, x) g[\phi(t, \cdot)](x) d t d x .
$$

Gathering (3.16), (3.18) and (3.20) in $T_{1}+T_{1}+T_{13}=0$ leads to

$$
\begin{aligned}
& \int_{0}^{\infty} \int_{\mathbb{R}} u(t, x) \partial_{t} \phi(t, x) d t d x+\int_{0}^{\infty} \int_{\mathbb{R}} f(u(t, x)) \partial_{x} \phi(t, x) d t d x \\
&-\int_{0}^{\infty} \int_{\mathbb{R}} u(t, x) g[\phi(t, \cdot)](x) d t d x=\int_{\mathbb{R}} u_{0}(x) \phi(0, x) d x .
\end{aligned}
$$

This proves that $u$ is a weak solution to (1.1). If $\lambda>1$, this weak solution is in fact the unique solution in the sense of Duhamel's formula, and thus also the unique smooth strong solution (see 14), and the proof is complete. If $\lambda \leq 1$, we must modify the preceding reasoning to show, using (2.10), that $u$ is an entropy solution to (1.1).

Under assumptions (2.3) and (2.4), (2.2) can be written as

$$
\begin{aligned}
u_{i}^{n+1} & =u_{i}^{n}-\frac{\delta t}{\delta x} F\left(u_{i}^{n}, u_{i+1}^{n}\right)+\frac{\delta t}{\delta x} F\left(u_{i-1}^{n}, u_{i}^{n}\right)-\delta t g^{\delta x}\left[u^{n+1}\right]_{i} \\
& =H\left(u_{i-1}^{n}, u_{i}^{n}, u_{i+1}^{n}\right)-\delta t g^{\delta x}\left[u^{n+1}\right]_{i}
\end{aligned}
$$

where $H$ is non-decreasing with respect to each of its variables on $\left[\inf _{\mathbb{R}} u_{0}, \sup _{\mathbb{R}} u_{0}\right]^{3}$ and, for all $\kappa \in\left[\inf _{\mathbb{R}} u_{0}, \sup _{\mathbb{R}} u_{0}\right], H(\kappa, \kappa, \kappa)=\kappa$. Denoting $a \top b=\max (a, b)$, we have in particular $H\left(u_{i-1}^{n}, u_{i}^{n}, u_{i+1}^{n}\right) \leq H\left(u_{i-1}^{n} \top \kappa, u_{i}^{n} \top \kappa, u_{i+1}^{n} \top \kappa\right)$ and $\kappa \leq$ $H\left(u_{i-1}^{n} \top \kappa, u_{i}^{n} \top \kappa, u_{i+1}^{n} \top \kappa\right)$ and we deduce, examining separately the cases $u_{i}^{n+1} \leq \kappa$ and $u_{i}^{n+1}>\kappa$,

$$
u_{i}^{n+1} \top \kappa \leq H\left(u_{i-1}^{n} \top \kappa, u_{i}^{n} \top \kappa, u_{i+1}^{n} \top \kappa\right)-\mathbf{1}_{\kappa, \infty[}\left(u_{i}^{n+1}\right) \delta t g^{\delta x}\left[u^{n+1}\right]_{i} .
$$

Similarly, if $a \perp b=\min (a, b)$, then

$$
u_{i}^{n+1} \perp \kappa \geq H\left(u_{i-1}^{n} \perp \kappa, u_{i}^{n} \perp \kappa, u_{i+1}^{n} \perp \kappa\right)-\mathbf{1}_{]-\infty, \kappa[}\left(u_{i}^{n+1}\right) \delta t g^{\delta x}\left[u^{n+1}\right]_{i}
$$

and therefore

$$
\begin{aligned}
u_{i}^{n+1} \top \kappa-u_{i}^{n+1} \perp \kappa \leq & H\left(u_{i-1}^{n} \top \kappa, u_{i}^{n} \top \kappa, u_{i+1}^{n} \top \kappa\right)-H\left(u_{i-1}^{n} \perp \kappa, u_{i}^{n} \perp \kappa, u_{i+1}^{n} \perp \kappa\right) \\
& -\left(\mathbf{1}_{\kappa, \infty}\left[\left(u_{i}^{n+1}\right)-\mathbf{1}_{]-\infty, \kappa[}\left(u_{i}^{n+1}\right)\right) \delta t g^{\delta x}\left[u^{n+1}\right]_{i} .\right.
\end{aligned}
$$

Defining $\eta_{\kappa}(s)=|s-\kappa|=s \top \kappa-s \perp \kappa$, we have $\eta_{\kappa}^{\prime}(s)=\mathbf{1}_{\kappa, \infty[}(s)-\mathbf{1}_{]-\infty, \kappa[}(s)$ (this selects the subdifferential of $\eta_{\kappa}$ equal to 0 at $\left.s=0\right)$ and the definition of $H$ thus leads to

$$
\begin{aligned}
& \frac{\delta x}{\delta t}\left(\eta_{\kappa}\left(u_{i}^{n+1}\right)-\eta_{\kappa}\left(u_{i}^{n}\right)\right)+\left(F\left(u_{i}^{n} \top \kappa, u_{i+1}^{n} \top \kappa\right)-F\left(u_{i}^{n} \perp \kappa, u_{i+1}^{n} \perp \kappa\right)\right) \\
& \quad-\left(F\left(u_{i-1}^{n} \top \kappa, u_{i}^{n} \top \kappa\right)-F\left(u_{i-1}^{n} \perp \kappa, u_{i}^{n} \perp \kappa\right)\right)+\delta x \eta_{\kappa}^{\prime}\left(u_{i}^{n+1}\right) g^{\delta x}\left[u^{n+1}\right]_{i} \leq 0 .
\end{aligned}
$$


Taking $r>0$, applying (2.10) and using (3.2) for $g_{\lambda, r}^{\delta x}$ (which satisfies the assumptions of Lemma 3.1), we find

$$
\begin{aligned}
\frac{\delta x}{\delta t}\left(\eta_{\kappa}\left(u_{i}^{n+1}\right)-\eta_{\kappa}\left(u_{i}^{n}\right)\right) & +\left(F\left(u_{i}^{n} \top \kappa, u_{i+1}^{n} \top \kappa\right)-F\left(u_{i}^{n} \perp \kappa, u_{i+1}^{n} \perp \kappa\right)\right) \\
& -\left(F\left(u_{i-1}^{n} \top \kappa, u_{i}^{n} \top \kappa\right)-F\left(u_{i-1}^{n} \perp \kappa, u_{i}^{n} \perp \kappa\right)\right) \\
+ & \delta x g_{\lambda, r}^{\delta x}\left[\eta_{\kappa}\left(u^{n+1}\right)\right]_{i}+\delta x \eta_{\kappa}^{\prime}\left(u_{i}^{n+1}\right) g_{0, r}^{\delta x}\left[u^{n+1}\right]_{i} \leq 0 .
\end{aligned}
$$

These inequalities (for all $r>0$ ) are discrete versions of the entropy inequalities for (1.1), and it is quite straightforward to deduce from them that the limit $u$ of $u_{\delta t, \delta x}$ satisfies the entropy inequalities for (1.1). Indeed, taking a non-negative $\phi \in C_{c}^{2}\left(\left[0, \infty[\times \mathbb{R})\right.\right.$, defining $\Phi_{i}^{n}$ from $\phi$ as before, multiplying (3.21) by $\delta t \Phi_{i}^{n}$ and summing on $n$ and $i$, we obtain $T_{4}+T_{5}+T_{6}+T_{7} \leq 0$, where

- T四 is T with $u_{i}^{n}$ replaced by $\eta_{k}\left(u_{i}^{n}\right)$,

- T $T_{5}$ is T2 with $F\left(u_{i}^{n}, u_{i+1}^{n}\right)$ replaced by $F\left(u_{i}^{n} \top \kappa, u_{i+1}^{n} \top \kappa\right)-F\left(u_{i}^{n} \perp \kappa, u_{i+1}^{n} \perp \kappa\right)$ and $F\left(u_{i-1}^{n}, u_{i}^{n}\right)$ replaced by $F\left(u_{i-1}^{n} \top \kappa, u_{i}^{n} \top \kappa\right)-F\left(u_{i-1}^{n} \perp \kappa, u_{i}^{n} \perp \kappa\right)$,

- T西 is T with $g^{\delta x}$ replaced by $g_{\lambda, r}^{\delta x}$ and $u^{n+1}$ replaced by $\eta_{k}\left(u^{n+1}\right)$

and

$$
T^{7}=\sum_{n \geq 0} \delta t \sum_{i \in \mathbb{Z}} \delta x \eta_{\kappa}^{\prime}\left(u_{i}^{n+1}\right) g_{0, r}^{\delta x}\left[u^{n+1}\right]_{i} \Phi_{i}^{n} .
$$

Using the same techniques as in the study of convergence of $T_{1}, T_{2}$ and $T_{3}$, the strong convergence of $u_{\delta t, \delta x}$ to $u$ allows us to see that, as $\delta t$ and $\delta x$ tend to 0 ,

$$
\begin{gathered}
T_{\text {T }} \rightarrow-\int_{0}^{\infty} \int_{\mathbb{R}} \eta_{\kappa}(u(t, x)) \partial_{t} \phi(t, x) d t d x-\int_{\mathbb{R}} \eta_{\kappa}\left(u_{0}(x)\right) \phi(0, x) d x, \\
T_{[5} \rightarrow-\int_{0}^{\infty} \int_{\mathbb{R}}(f(u(t, x) \top \kappa)-f(u(t, x) \perp \kappa)) \partial_{x} \phi(t, x) d t d x
\end{gathered}
$$

and

$$
T_{[6} \rightarrow \int_{0}^{\infty} \int_{\mathbb{R}} \eta_{\kappa}(u(t, x)) g_{\lambda, r}[\phi(t, \cdot)](x) d t d x .
$$

Regarding Th, we have

$$
T_{7}=\int_{0}^{\infty} \int_{\mathbb{R}} \eta_{\kappa}^{\prime}\left(u_{\delta t, \delta x}(t+\delta t, x)\right) V_{\delta t, \delta x}(t, x) \Phi_{\delta t, \delta x}(t, x) d t d x,
$$

where $V_{\delta t, \delta x}=g_{0, r}^{\delta x}\left[u^{n+1}\right]_{i}$ and $\Phi_{\delta t, \delta x}=\Phi_{i}^{n}$ on $[n \delta t,(n+1) \delta t[\times[i \delta x,(i+1) \delta x[$ for all $n \geq 0$ and all $i \in \mathbb{Z}$. By (2.10), for all compact $Q$ and all $t \geq 0$, taking $n \geq 0$ such that $t \in\left[n \delta t,(n+1) \delta t\left[\right.\right.$, we have $\left\|V_{\delta t, \delta x}(t, \cdot)-g_{0, r}\left[u^{n+1}\right]\right\|_{L^{1}(Q)} \leq$ $\left\|u^{n+1}\right\|_{L^{\infty}(\mathbb{R})} \gamma_{r, Q}(\delta x) \leq\left\|u_{0}\right\|_{L^{\infty}(\mathbb{R})} \gamma_{r, Q}(\delta x)$. From the definition of $g_{0, r}$ and the convergence of $u_{\delta t, \delta x}$ to $u$ we see that the function defined by $g_{0, r}\left[u^{n+1}\right]$ on $[n \delta t,(n+$ 1) $\delta t\left[\times \mathbb{R}\right.$ converges to $g_{0, r}[u]$ in $L_{\text {loc }}^{1}([0, \infty[\times \mathbb{R})$, and we therefore deduce that, as $\delta t$ and $\delta x$ go to $0, V_{\delta t, \delta x}$ also converges to $g_{0, r}[u]$ in $L_{\text {loc }}^{1}([0, \infty[\times \mathbb{R})$. The convergence of $u_{\delta t, \delta x}$ to $u$ in $L_{\text {loc }}^{1}\left(\left[0, \infty[\times \mathbb{R})\right.\right.$ shows that $u_{\delta t, \delta x}(\cdot+\delta t, \cdot)$ also converges in this space to $u$ and thus, up to a subsequence, a.e. on $] 0, \infty[\times \mathbb{R}$; but, for a.e. $\kappa \in \mathbb{R}$, the measure of $\{(t, x) \in] 0, \infty[\times \mathbb{R}, u(t, x)=\kappa\}$ vanishes and, since $\eta_{\kappa}^{\prime}$ is continuous on $\mathbb{R} \backslash\{\kappa\}$, we have, for such $\kappa, \eta_{\kappa}^{\prime}\left(u_{\delta t, \delta x}(\cdot+\delta t, \cdot)\right) \rightarrow \eta_{\kappa}^{\prime}(u)$ a.e. on $] 0, \infty[\times \mathbb{R}$. Combined with the fact that $\left|\eta_{\kappa}^{\prime}\right| \leq 1$, the convergence of $V_{\delta t, \delta x}$ to $g_{0, r}[u]$ in $L_{\text {loc }}^{1}$, 
the uniform convergence of $\Phi_{\delta t, \delta x}$ to $\phi$ and the fact that the support of $\Phi_{\delta t, \delta x}$ stays in a compact subset of $[0, \infty[\times \mathbb{R}$, this allows us to pass to the limit in (3.25) to find

$$
T_{\text {T }} \rightarrow \int_{0}^{\infty} \int_{\mathbb{R}} \eta_{\kappa}^{\prime}(u(t, x)) g_{0, r}[u(t, \cdot)](x) \phi(t, x) d t d x .
$$

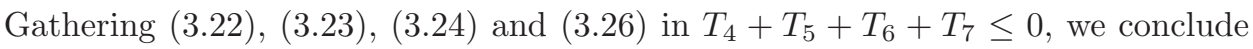
that

$$
\begin{aligned}
\int_{0}^{\infty} & \int_{\mathbb{R}} \eta_{\kappa}(u(t, x)) \partial_{t} \phi(t, x) d t d x \\
& +\int_{0}^{\infty} \int_{\mathbb{R}}(f(u(t, x) \top \kappa)-f(u(t, x) \perp \kappa)) \partial_{x} \phi(t, x) d t d x \\
& -\int_{0}^{\infty} \int_{\mathbb{R}} \eta_{\kappa}(u(t, x)) g_{\lambda, r}[\phi(t, \cdot)](x) d t d x \\
& -\int_{0}^{\infty} \int_{\mathbb{R}} \eta_{\kappa}^{\prime}(u(t, x)) g_{0, r}[u(t, \cdot)](x) \phi(t, x) d t d x \\
& +\int_{\mathbb{R}} \eta_{\kappa}\left(u_{0}(x)\right) \phi(0, x) d x \geq 0,
\end{aligned}
$$

where we recall that $\eta_{\kappa}(s)=|s-\kappa|$ and $\eta_{\kappa}^{\prime}(s)=\mathbf{1}_{\kappa, \infty[}(s)-\mathbf{1}_{]-\infty, \kappa[}(s)$. This inequality has been proved up to now only for almost every $\kappa \in \mathbb{R}$; but for any $\kappa \in \mathbb{R}$ we can choose $\left(\kappa_{m}\right)_{m>1}$ and $\left(\widetilde{\kappa}_{m}\right)_{m \geq 1}$ such that (3.27) is valid with $\kappa=\kappa_{m}$ and $\kappa=\widetilde{\kappa}_{m}$ and such that $\kappa_{m} \nearrow \kappa$ and $\widetilde{\kappa}_{m} \searrow \kappa$, and we have then $\frac{1}{2}\left(\eta_{\kappa_{m}}+\eta_{\widetilde{\kappa}_{m}}\right) \rightarrow \eta_{\kappa}$ and $\frac{1}{2}\left(\eta_{\kappa_{m}}^{\prime}+\eta_{\widetilde{\kappa}_{m}}^{\prime}\right) \rightarrow \eta_{\kappa}^{\prime}$ on $\mathbb{R}$ as $m \rightarrow \infty$, all these functions staying bounded on bounded subsets of $\mathbb{R}$; we can therefore take the mean value of (3.27) applied to $\kappa_{m}$ and $\widetilde{\kappa}_{m}$ and let $m \rightarrow \infty$ to see that (3.27) is also satisfied with $\kappa$. This shows that $u$ is the unique entropy solution to (1.1) (see [1) and concludes the proof.

Remark 3.10. We could as well consider the multi-dimensional form of (1.1) (i.e. with $N$ space dimensions instead of one); on Cartesian grids, the adaptation of the preceding reasoning is straightforward; on unstructured grids, however, the schemes for scalar conservation laws are not necessarily TVD (total variation decreasing) and it is therefore not possible to directly prove Corollary 3.8 , even if $u_{0} \in B V\left(\mathbb{R}^{N}\right)$, we have then to rely on the techniques sketched in Remark 3.9 .

\section{IMPLEMENTATION OF THE NUMERICAL METHOD}

\subsection{A few words on the resolution procedure.}

4.1.1. Example of $g^{\delta x}$. A space step $\delta x>0$ being chosen, Formula (1.2) makes it easy to write a discretization of $g$ : we approximate each integral sign using a basic quadrature rule on the mesh $\left(\left[j \delta x,(j+1) \delta x[)_{j \in \mathbb{Z}}\right.\right.$ (for example the right rectangles for $z>0$ and the left rectangles for $z<0$; this avoids the singularity of $1 /|z|^{1+\lambda}$ at $z=0$ and preserves the symmetry between $z>0$ and $z<0$ ) and we use a finite difference approximation of the derivative (for example a centered one). However, such an approximation would use all the $\left(v_{j}\right)_{j \in \mathbb{Z}}$ in order to compute $g^{\delta x}[v]_{i}$; in practical applications, the considered functions are usually constant near $-\infty$ and $+\infty$ : it is therefore safe to assume this when discretizing $g$ and to use the mesh $\left(\left[j \delta x,(j+1) \delta x[)_{j \in \mathbb{Z}}\right.\right.$ only up to $|z|=J_{\delta x} \delta x$ (for some integer $J_{\delta x}$ such that 
$J_{\delta x} \delta x \rightarrow+\infty$ as $\delta x \rightarrow 0$ ), approximating the remaining parts with two unbounded space steps $\left.]-\infty,-J_{\delta x} \delta x\right]$ and $\left[J_{\delta x} \delta x,+\infty[\right.$. This leads to

$$
\begin{aligned}
g^{\delta x}[v]_{i}= & -c(\lambda) \sum_{0<|j| \leq r / \delta x} \delta x \frac{v_{i+j}-v_{i}-\frac{v_{i+1}-v_{i-1}}{2 \delta x} j \delta x}{|j \delta x|^{1+\lambda}} \\
& -c(\lambda) \sum_{r / \delta x<|j| \leq J_{\delta x}} \delta x \frac{v_{i+j}-v_{i}}{|j \delta x|^{1+\lambda}} \\
& -c(\lambda) \frac{v_{i-J_{\delta x}-1}-v_{i}}{\lambda\left(J_{\delta x} \delta x\right)^{\lambda}}-c(\lambda) \frac{v_{i+J_{\delta x}+1}-v_{i}}{\lambda\left(J_{\delta x} \delta x\right)^{\lambda}}
\end{aligned}
$$

But $\sum_{0<|j| \leq r / \delta x} \frac{j}{|j \delta x|^{1+\lambda}}=0$ by symmetry, and we can in fact drop the discretization of the derivative:

$$
\begin{aligned}
g^{\delta x}[v]_{i}= & -c(\lambda) \sum_{0<|j| \leq r / \delta x} \delta x \frac{v_{i+j}-v_{i}}{|j \delta x|^{1+\lambda}} \\
(4.2) \quad & -c(\lambda) \sum_{r / \delta x<|j| \leq J_{\delta x}} \delta x \frac{v_{i+j}-v_{i}}{|j \delta x|^{1+\lambda}}-c(\lambda) \frac{v_{i-J_{\delta x}-1}-v_{i}}{\lambda\left(J_{\delta x} \delta x\right)^{\lambda}}-c(\lambda) \frac{v_{i+J_{\delta x}+1}-v_{i}}{\lambda\left(J_{\delta x} \delta x\right)^{\lambda}} \\
(4.3)= & -c(\lambda) \sum_{0<|j| \leq J_{\delta x}} \delta x \frac{v_{i+j}-v_{i}}{|j \delta x|^{1+\lambda}}-c(\lambda) \frac{v_{i-J_{\delta x}-1}-v_{i}}{\lambda\left(J_{\delta x} \delta x\right)^{\lambda}}-c(\lambda) \frac{v_{i+J_{\delta x}+1}-v_{i}}{\lambda\left(J_{\delta x} \delta x\right)^{\lambda}} .
\end{aligned}
$$

This dropping of the discretization of the derivative is in concordance with the reason behind the existence of $\phi^{\prime}(x)$ in (1.2); in fact, $g$ is essentially the principal value of $(|\cdot| 1-\lambda)^{\prime \prime}$ and $g[\phi]$ is therefore the limit as $\varepsilon \rightarrow 0$ of $-c(\lambda) \int_{|z| \geq \varepsilon} \frac{\phi(x+z)-\phi(x)}{|z|^{1+\lambda}} d z$ (see [15]); the term $\phi^{\prime}(x) z$ is introduced on ] - r, $r$ [ because, by symmetry, it does not modify this integral sign but makes it possible to write the limit as $\varepsilon \rightarrow 0$ as an integral sign on $\mathbb{R}$. In the framework of numerical analysis, there is no such question of principal value and integrability at 0 , and the disappearance of the discrete derivative is therefore not surprising.

It is easy to prove the first properties (2.5)-(2.8) for $g^{\delta x}$ defined by (4.3). Indeed, if $\lim _{k \rightarrow \infty} v_{i_{k}}=\sup _{j} v_{j}$, then, for all $\varepsilon>0$ and for $k$ large enough, $v_{i_{k}+j}-v_{i_{k}} \leq \varepsilon$ for all $j \in \mathbb{Z}$ and thus

$$
g^{\delta x}[v]_{i_{k}} \geq-c(\lambda) \varepsilon \sum_{0<|j| \leq J_{\delta x}} \delta x \frac{1}{|j \delta x|^{1+\lambda}}-\frac{2 c(\lambda) \varepsilon}{\lambda\left(J_{\delta x} \delta x\right)^{\lambda}}=-C(\delta x) \varepsilon
$$

and (2.6) is obtained by taking the $\liminf _{k \rightarrow \infty}$ of this inequality and then letting $\varepsilon \rightarrow 0$. The linearity (2.5), the invariance by translation (2.7) and the dependence on a finite number of values (2.8) are obviously satisfied. The proof of Properties (2.9) and (2.10) is way more technical and is therefore given in the appendix (Lemma 5.1).

4.1.2. Choice of the parameters. The practical implementation of the scheme (2.1) -(2.2) requires us to make some choices of truncation parameters. First of all, we cannot obviously compute the approximate solution on the whole of $[0, \infty[\times \mathbb{R}$; we have to select a bounded domain on which we intend to obtain the solution: assume that this domain is $[0, T] \times[-D, D]$. To simplify the presentation, we also assume that $\delta t=T / N_{\delta t}$ and $\delta x=D / N_{\delta x}$ for some integers $N_{\delta t}$ and $N_{\delta x}$.

If we forget for a moment the operator $g^{\delta x}$ in (2.2), we notice that the calculation of $\left(u_{i}^{n+1}\right)_{|i| \leq N_{\delta x}}$ (in order to obtain the approximate solution at time step $n+1$ 
on $[-D, D])$ necessitates knowing $\left(u_{i}^{n}\right)_{|i| \leq N_{\delta x}+1}\left(\right.$ or $\left(u_{i}^{n}\right)_{|i| \leq N_{\delta x}+2}$ in the case of 4points numerical fluxes instead of 2-points fluxes). Hence, the hyperbolic part of the scheme imposes to begin at $t=0$ with the indexes $|i| \leq N_{\delta x}+N_{\delta t}$ (or $N_{\delta x}+2 N_{\delta t}$ in the case of the 4-points scheme) in order to obtain the approximate solution at time $t=T$ on $[-D, D]$; this is the discrete counterpart of the well-known finite speed propagation of the scalar conservation laws.

But we must also consider the operator $g^{\delta x}$, which makes of the scheme a nontrivial infinite linear system. The proof of Lemma 3.3 however gives a way to approximate the solution to (2.2): $h_{i}$ being the right-hand side of (3.10) and $\alpha$ being equal to $\delta t$, an approximation of $\left(u_{i}^{n+1}\right)_{i \in \mathbb{Z}}$ is given by the solution to (3.7) for $m$ "large enough" ... but which $m$ ? It is not obvious to give an analytical answer to this question: it is possible, from (4.3), to estimate the convergence as $m \rightarrow \infty$ of the solution of (3.7) to the solution of (3.4); however, this general estimate is very slow (of order $\xi_{\delta x}^{m / J_{\delta x}}$ for some $\xi_{\delta x}<1$ ) and imposing $m$ using this error bound leads to unreasonable values. The same holds for the choice of $J_{\delta x}$ in the definition of $g_{\delta x}$ : it is easy to see that the difference between $g^{\delta x}$ defined by (4.3) and the same expression with an infinite series $\left(J_{\delta x}=+\infty\right)$ is of order $\|v\|_{l \infty(\mathbb{Z})}\left(J_{\delta x} \delta x\right)^{-\lambda}$ and thus, if we take $J_{\delta x}=\frac{\epsilon^{-1 / \lambda}}{\delta x}$, that the error, in the definition of $g^{\delta x}$, due to the truncation of the sum at $J_{\delta x}$ is of order at most $\epsilon$. However, the value thus chosen for $J_{\delta x}$ is not reasonable, especially if $\lambda$ is small. These general findings are in concordance with the estimate on the infinite speed propagation phenomenon of (1.1): it is proved in [1] that the influence of $u_{0}(x)$ on $u(1, y)$ decreases as $|x-y|^{-\lambda}$ (i.e. very slowly).

However, in practical situations, things behave much better than the preceding reasoning might let one believe (partly because the above bounds are quite rough; partly because the considered initial conditions are not any kind of function). Consider for example $T=0.5, D=1, \lambda=0.5$, a Burgers flux $f(s)=s^{2} / 2$ and a Riemann initial condition $u_{0}(x)=1$ if $x<0$ and $u_{0}(x)=-1$ if $x>0$ (we also use a 4-points MUSCL method based on the Godunov numerical flux, see [17, instead of a simple 2-points flux in (2.2)). Due to the hyperbolic part of the equation, we compute the solution for at least the indexes $|i| \leq N_{\delta x}+2 N_{\delta t}$, and it seems wise to take this value as a lower bound for the choice of $m$ in (3.7) (in order that the non-local operator influences all the terms coming from the hyperbolic part of the equation). To understand if a higher value of $m$ can improve the precision of the approximate solution, we show in Table 1 for various values of $N_{\delta t}$ and $N_{\delta x}$ (all satisfying the CFL condition associated with the MUSCL scheme), the difference between these solutions computed with the values $m=N_{\delta x}+2 N_{\delta t}$ and $m=3\left(N_{\delta x}+2 N_{\delta t}\right)$ (and in either case for $J_{\delta x}$ large enough to have a minimal interference): the very small difference between the two solutions shows that the choice $m=N_{\delta}+2 N_{\delta x}$ is sufficient to obtain, in most cases, a good approximate solution to the scheme.

As for $J_{\delta x}$, a minimal value appears to be $2 m$ in order that, when solving (3.7), the computation of $g^{\delta x}\left[v^{m}\right]_{i}$ takes into account all the $\left(v_{j}^{m}\right)_{j \in \mathbb{Z}}$ which are influenced by $g^{\delta x}$ in this system of equations. Here again, there is in fact little gain to be found in using a much larger value for $J_{\delta x}$ than this estimated minimum, as shown by Table 2 (in which we present the $L^{\infty}$ difference of the solutions computed with $m=N_{\delta x}+2 N_{\delta t}$ and either $J_{\delta x}=2 m$ or $J_{\delta x}=6 m$ ). Fixing $J_{\delta x}=2 m$ seems sufficient to obtain acceptable numerical approximations. 
TABLE 1. $L^{\infty}$ difference between the approximate solutions computed with $m=N_{\delta t}+2 N_{\delta x}$ and with $m=3\left(N_{\delta t}+2 N_{\delta x}\right)$ (and $J_{\delta x}=4 m$ in either case).

\begin{tabular}{|c|c|c|c|}
\hline$N_{\delta x}$ & $N_{\delta t}$ & $m$ & $L^{\infty}$ difference \\
\hline 50 & 100 & 250 and 750 & $2.02 \mathrm{E}-4$ \\
\hline 100 & 200 & 500 and 1500 & $2.05 \mathrm{E}-4$ \\
\hline 150 & 300 & 750 and 2250 & $2.06 \mathrm{E}-4$ \\
\hline
\end{tabular}

TABLE 2. $L^{\infty}$ difference between the approximate solutions computed with $J_{\delta x}=2 m$ and with $J_{\delta x}=6 m$ (and $m=N_{\delta}+2 N_{\delta x}$ in either case).

\begin{tabular}{|c|c|c|c|}
\hline$N_{\delta x}$ & $N_{\delta t}$ & $J_{\delta x}$ & $L^{\infty}$ difference \\
\hline 50 & 100 & 500 and 1500 & $1.76 \mathrm{E}-5$ \\
\hline 100 & 200 & 1000 and 3000 & $8.82 \mathrm{E}-6$ \\
\hline 150 & 300 & 1500 and 4500 & $5.88 \mathrm{E}-6$ \\
\hline
\end{tabular}

Notice that, once $m$ and $J_{\delta x}$ are chosen, we know exactly which indexes are to be considered in the implementation: the indexes $|i| \leq m+J_{\delta x}+1$ (this can be seen from (3.7), since the computation of $g^{\delta x}\left[v^{m}\right]_{i}$ for all $|i| \leq m$ uses only $\left.\left(v_{j}^{m}\right)|j| \leq m+J_{\delta x}+1\right)$.

4.1.3. Efficient numerical computation of the solution. Once the truncation parameters $m$ and $J_{\delta x}$ are chosen, computing an approximate solution to the scheme requires solving the following systems of the kind (3.7):

$$
\forall i \in \mathbb{Z}: u_{i}^{n+1}+\delta t g^{\delta x}\left[u^{n+1}\right]_{i} \mathbf{1}_{[-m, m]}(i)=h_{i}^{n},
$$

where $h^{n}$ is obtained by an iteration of the scheme for the pure scalar conservation law, i.e. $h_{i}^{n}=u_{i}^{n}+\frac{\delta t}{\delta x} F\left(u_{i-1}^{n}, u_{i}^{n}\right)-\frac{\delta t}{\delta x} F\left(u_{i}^{n}, u_{i+1}^{n}\right)$. This system imposes $u_{i}^{n+1}=h_{i}^{n}$ for $|i|>m$; defining then $v=\left(h_{i}^{n} \mathbf{1}_{\mathbb{Z} \backslash[-m, m]}(i)\right)_{|i| \in \mathbb{Z}}$ and $W=\left(u_{i}^{n+1}\right)_{|i| \leq m}$, (4.4) reduces to a square system of size $2 m+1$ on $W$ :

$$
W+\delta t G^{\delta x} W=\left(h_{i}^{n}-\delta t g^{\delta x}[v]_{i}\right)_{|i| \leq m},
$$

in which the matrix $G^{\delta x}$ comes from $g^{\delta x}\left(G^{\delta x} W=\left(g^{\delta x}[\widetilde{W}]_{i}\right)_{|i| \leq m}\right.$ with $\widetilde{W}_{i}=W_{i}$ if $|i| \leq m$ and $\widetilde{W}_{i}=0$ if $\left.|i|>m\right)$. It is easy to see from the definition of $g^{\delta x}$ that $\bar{G}^{\delta x}$ is a symmetric semi-definite positive (it is diagonal-dominant) Toeplitz matrix, and thus that the matrix $I+\delta t G^{\delta x}$ of (4.5) is symmetric definite positive Toeplitz; solving this system can therefore be done in an extremely fast way by using a preconditioned Conjugate Gradient method and multiplication algorithms coming from the FFT framework (see [12, 25] and also 22 for a possible adaptation to "more local" operators). Moreover, because of the Toeplitz form of $g^{\delta x}$, it is also possible to use FFT-based algorithms to achieve a very fast computation of the right-hand side of (4.5).

Hence, the scheme (2.1) - 2.2 is not only a proper theoretical approximation of (1.1), but also a very efficient one in terms of computational cost. We now give some numerical results to show that this scheme also provides nice practical approximations of solutions to fractal conservation laws. 
4.2. Numerical results. In the following numerical tests, we consider a Burgers flux $f(s)=s^{2} / 2$ and, in order to avoid introducing too much numerical diffusion, we use a 4-points MUSCL method based on the Godunov flux [17] to compute the numerical fluxes associated with $f$. Except in Section 4.2.3, we present snapshots of the approximate solution 2 at time $T=0.5$ on the domain $[-1,1]$, computed with a space step $\delta x=6.67 \times 10^{-3}$ and a time step $\delta t=1.67 \times 10^{-3}$ (with our choices of initial conditions, these values satisfy the CFL condition associated with the MUSCL method); we use $g^{\delta x}$ given by (4.3), the parameters $m$ and $J_{\delta x}$ being chosen according to the discussion in the preceding section $(\delta x$ and $\delta t$ correspond to the choices $N_{\delta x}=150$ and $N_{\delta t}=300$, so $m=N_{\delta x}+2 N_{\delta t}=750$ and $\left.J_{\delta x}=2 m=1500\right)$.

Note that $\delta x=6.67 \times 10^{-3}$ and $\delta t=1.67 \times 10^{-3}$ are not very small steps; thanks to the algorithms mentioned in Section 4.1 .3 , each of the following numerical tests only takes a few seconds on a personal computer, and it would not be a strong computational issue to reduce the size of the time-space grid. We choose to present the results using these values of $\delta x$ and $\delta t$ in order to show that the numerical outputs of the scheme are quite good even without using a very fine grid.

4.2.1. Shock preservation and creation. If $\lambda>1$, the solution to (1.1) is $C^{\infty}$-regular for any bounded initial data (see [14). If $\lambda<1$, however, it is proved in 3 that the diffusion properties of $g$ are not always strong enough, when in the presence of a Burgers flux, to smoothen discontinuous initial data; moreover, in this situation, even $C^{\infty}$-regular initial data can give rise to discontinuous solutions.

These two different behaviors (smoothing or shock preservation) with respect to a discontinuous initial condition are illustrated in Figure 1 (in which the initial condition is of Riemann type: $u_{0}(x)=1$ if $x<0$ and $u_{0}(x)-1$ if $x>0$ ). The figure clearly shows that the solution corresponding to $\lambda=0.3$ presents a shock at $x=0$, whereas the solution for $\lambda=1.5$ is smooth.

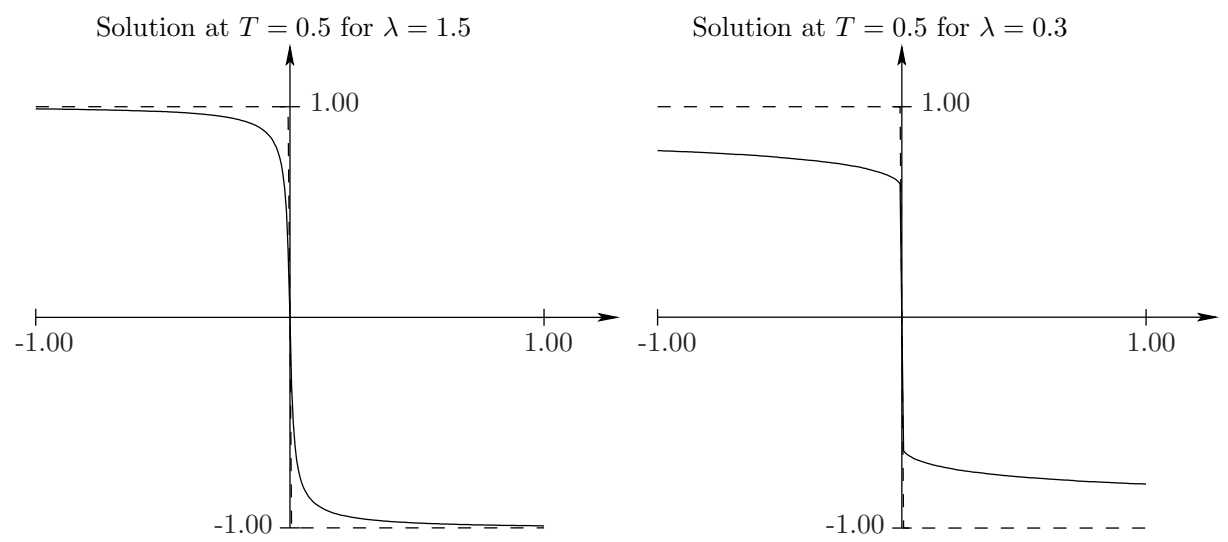

FiguRE 1. Smoothing effect for $\lambda>1$ and preservation of shock for $\lambda<1$ (the dotted line is the common initial condition of these tests).

The phenomenon of shock creation if $\lambda<1$ is shown in Figure 2 in this test, we take a kind of initial data, which, although Lipschitz continuous, ensures that the

\footnotetext{
${ }^{2}$ Or rather of affine interpolates of the constant-by-parts approximate solutions (these affine interpolates also converge to the solution of (1.1).
} 
solution develops a shock in finite time (see [3] $): u_{0}(x)=\min (1, \max (-3 x,-1))\left(u_{0}\right.$ is in fact piecewise linear, with a strong negative slope around 0 which provokes the creation of a shock; we could have smoothen $u_{0}$ around its slope discontinuities at $x=-1 / 3$ and $x=1 / 3$ without much changing the behavior of the solution).

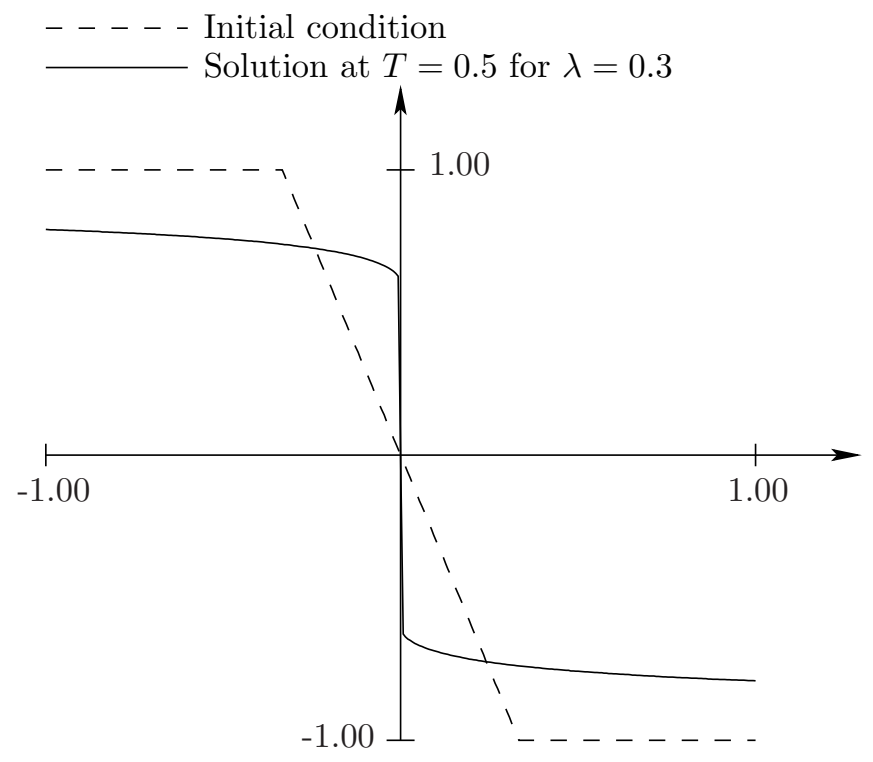

Figure 2. Creation of shock for $\lambda<1$.

4.2.2. Speeds of diffusion. Let us consider for a moment the pure fractal equation, i.e. $f=0$ in (1.1). It is known that, for any $\lambda \in] 0,2\left[\right.$, the solution to $\partial_{t} u+g[u]=0$ is regular. The diffusive effects of the operator $g$, which explains this regularizing effect, however depend on the value of $\lambda$; indeed, taking the Fourier transform of $\partial_{t} u+g[u]=0$ we see that $\partial_{t} \mathcal{F}(u)+|\xi|^{\lambda} \mathcal{F}(u)=0$ : thus, during the evolution, the larger $\lambda$, the more high frequencies are reduced and the less low frequencies are diffused. This property explains in particular the different behaviors in the presence of a Burgers flux with respect to shocks (Section 4.2.1), but is also illustrated, for the pure fractal equation, in Figure 3 the initial data used in this test $(-1$ if $x<0$, +1 if $x>0$ ) has mainly low frequencies and is globally less diffused for a higher $\lambda$, except around the discontinuity (high frequency) where the smoothing is stronger (the slope of the solution is smaller).

The presence of a flux can also interact with the different diffusive properties of $g$ for various $\lambda$. If, keeping the same non-decreasing discontinuous initial data, we add a Burgers flux (i.e. we consider (1.1) with $f(s)=s^{2} / 2$ ), then the hyperbolic part of the equation generates a rarefaction wave: the initial shock is transformed into a piecewise-linear solution; the high frequencies are therefore killed by the flux and it can be seen in Figure 4 that the behaviors of the solutions for various $\lambda$ no longer differ around the initial shock (in fact, from (1.2) we can see that $g$ vanishes on affine functions for any $\lambda$ ). The stronger diffusive effect for low $\lambda$ is however still perceptible in the zones of lower frequencies of the solution. 


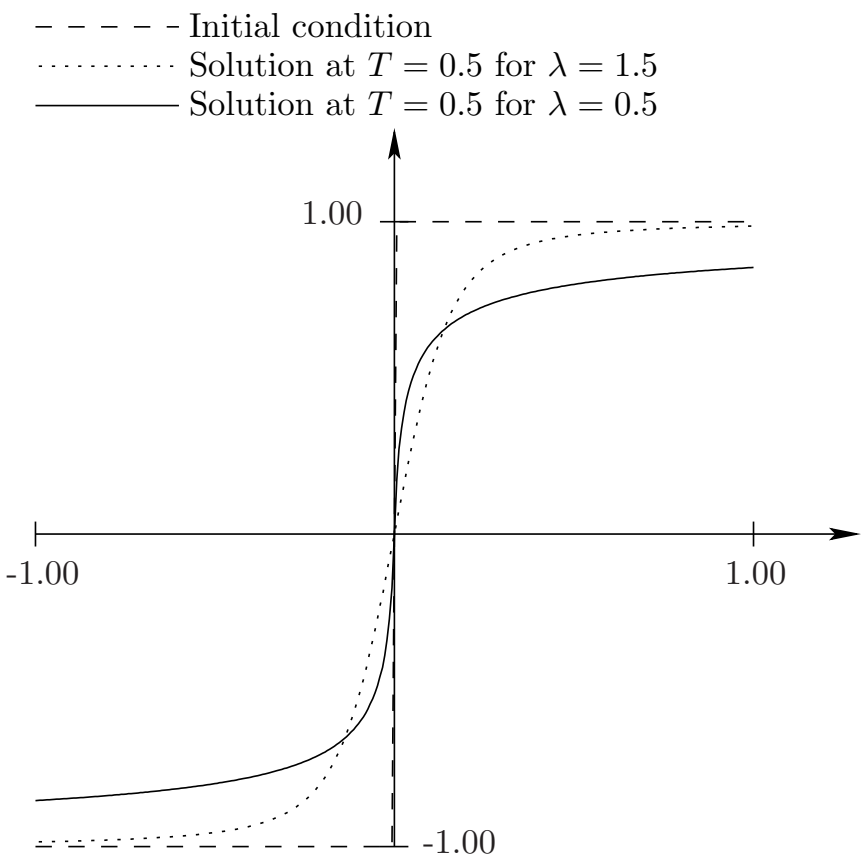

Figure 3. Solutions, for various $\lambda$ and an initial shock, to the pure fractal equation $\partial_{t} u+g[u]=0$.

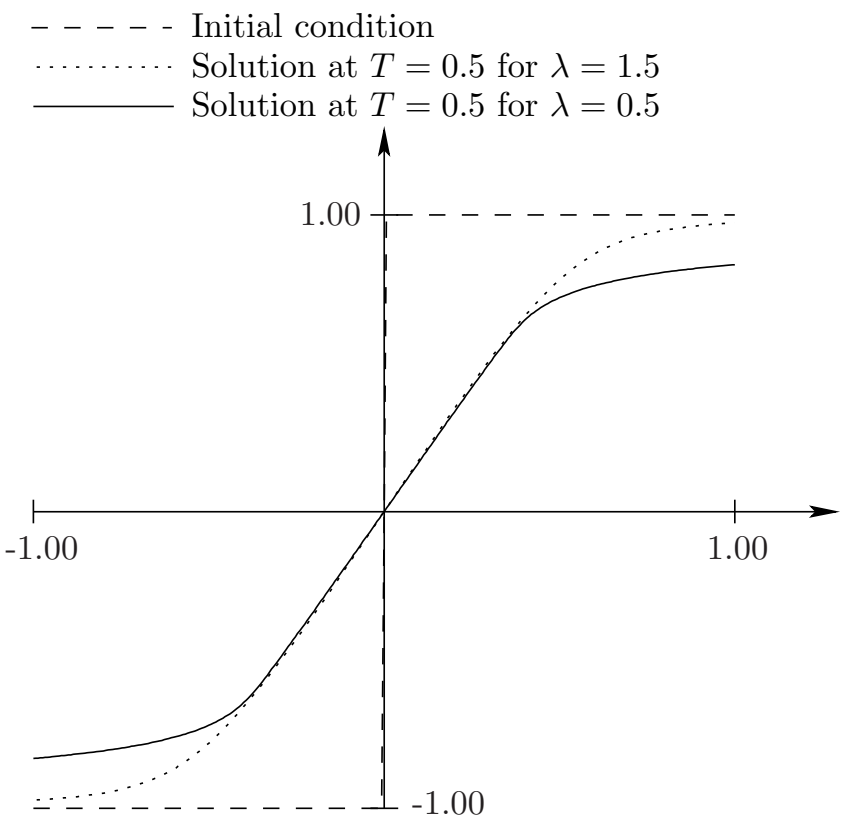

Figure 4. Solutions, for various $\lambda$, to (1.1) in the case of an initial data and a flux $f(s)=s^{2} / 2$ generating a rarefaction wave. 
4.2.3. Asymptotic behavior. In [7, the asymptotic behavior as $t \rightarrow \infty$ of the solution to $\partial_{t} v+\partial_{x}(f(v))+g[v]-\Delta v=0$ is studied; the addition, with respect to (1.1), of the Laplacian term provokes little disturbance in the long-time behavior of the solution, and the results of this reference are also valid for (1.1). Let us try to illustrate them with the help of the numerical scheme.

We take $\lambda=0.5$, and an initial data $u_{0}$ equal to 1 on $[-0.2,0.2]$ and to 0 elsewhere; the time-space domain of discretization is $[0,30] \times[-1,1]$ and, to avoid that the rarefaction wave and the shock generated by a Burgers flux for $u_{0}$ leave the domain of study, we reduce the strength of the flux by taking $f(s)=s^{2} / 6$. Denoting by $K(t, x)$ the kernel of $\partial_{t}+g=0$ (i.e. $\partial_{t} K(t, x)+g[K(t, \cdot)](x)=0$ and $K(t, \cdot) \rightarrow \delta_{0}$ as $\left.t \rightarrow 0\right)$, it is proved in [7] that $t^{1 / \lambda}\left[u(t)-K(t) * u_{0}\right] \rightarrow 0$ in $L^{\infty}(\mathbb{R})$ as $t \rightarrow \infty$.

Figures 5 and 6 illustrate this property, by showing on one side the functions $x \mapsto u(t, x)$ and $x \mapsto K(t) * u_{0}(x)$ for various times, and on the other side the plot in a log-log scale of the $L^{\infty}$-norm of $t^{1 / \lambda}\left[u(t)-K(t) * u_{0}\right]$ on $[-1,1]$ versus the time; the approximations of $u$ and $K * u_{0}$ (solution to (1.1) with $f=0$ ) used to draw these figures have been computed on $[0,30] \times[-1,1]$ using the numerical scheme with $N_{\delta x}=100$ and $N_{\delta t}=4000$. It is proved in [7] that the next term in the asymptotic expansion of $u(t)$ is of order $t^{-2 / \lambda}$, i.e. that $\left\|t^{1 / \lambda}\left[u(t)-K(t) * u_{0}\right]\right\|_{L^{\infty}(\mathbb{R})}=\mathcal{O}\left(t^{-1 / \lambda}\right)$ as $t \rightarrow \infty$, and the reference slope $t \mapsto t^{-1 / \lambda}$ in Figure 6 confirms this (see below regarding the change of behavior after $t=10$ ).

In fact, the second term in the asymptotics of $u(t)$ is known: it is proportional to $\partial_{x} K(t)$ (see [7); the numerical capture of this term is however quite challenging. Indeed, since $K(t, x)=t^{-1 / \lambda} K\left(1, t^{-1 / \lambda} x\right)$, we have $\partial_{x} K(t, x)=t^{-2 / \lambda} \partial_{x} K\left(1, t^{-1 / \lambda} x\right)$ and $\partial_{x} K(t)$ is of order $t^{-2 / \lambda}$ in $L^{\infty}(\mathbb{R})$ but, as $\partial_{x} K(1,0)=0$, its maximal absolute values are attained at points $x$ which go to $\pm \infty$ with $t^{1 / \lambda}$; restricted to $[-1,1], \partial_{x} K(t)$ is in fact of order $t^{-3 / \lambda}$ and can thus interact with a possible third (and yet unknown) term in the expansion; this behavior is in concordance with the acceleration of convergence which clearly appears in Figure [6 restricted to $[-1,1], t^{1 / \lambda}\left[u(t)-K(t) * u_{0}\right]$ seems to be asymptotically more of order $t^{-2 / \lambda}$ than $t^{-1 / \lambda}$. Numerically illustrating the second term in the asymptotics of $u(t)$ would therefore require approximating this solution on a large time-space scale (including the extremal values of $\partial_{x} K$ ) and with a very high degree of precision (so that the numerical error is negligible with respect to $t^{-2 / \lambda}$ ), which is beyond standard computational power.

Notice that this problem does not appear for the first term $K(t) * u_{0}$ in the asymptotic expansion: its maximum absolute value is of order $t^{-1 / \lambda}$ and is attained in $[-1,1]$ for all $t>0$; a reasonable approximation of $u(t)$ on $[-1,1]$ thus suffices to capture this term. This is shown in Figure 6, and also confirmed if we look at the relative $L^{\infty}$-error on $[-1,1]$ between $u(t)$ and $K(t) * u_{0}$ : for $t=1$, this computed error $\frac{\left\|u(t)-K(t) * u_{0}\right\|_{L} \infty([-1,1])}{\left\|K(t) * u_{0}\right\|_{L}^{\infty}([-1,1])}$ is around 0.63 , whereas it is around 0.047 for $t=10$ and around 0.0014 for $t=30$. We are thus confident that the numerical scheme really has captured the proximity of $u(t)$ and $K(t) * u_{0}$ for $t$ large, not only a small quantity due to the difference of two small functions. 


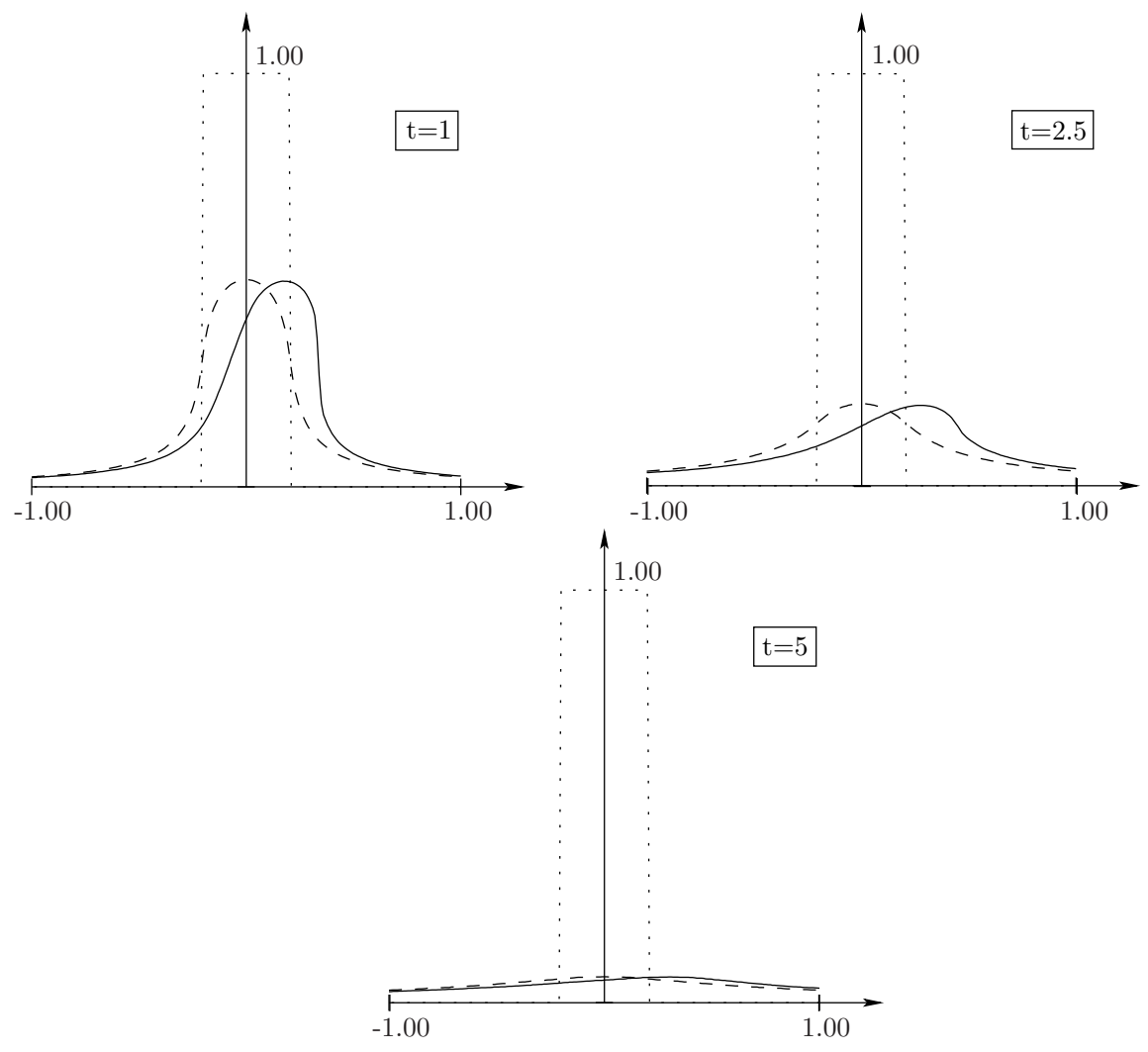

Figure 5. Initial condition $u_{0}$ (dotted line), $K(t) * u_{0}$ (dashed line) and $u(t)$ solution to $\partial_{t} u+\partial_{x}\left(u^{2} / 6\right)+g[u]=0$ (continuous line), for $\lambda=0.5$ and various times.

4.3. About the explicit scheme. The explicit form of the scheme consists in replacing (2.2) with

$$
\forall n \geq 0, \forall i \in \mathbb{Z}: \frac{\delta x}{\delta t}\left(u_{i}^{n+1}-u_{i}^{n}\right)+F\left(u_{i}^{n}, u_{i+1}^{n}\right)-F\left(u_{i-1}^{n}, u_{i}^{n}\right)+\delta x g^{\delta x}\left[u^{n}\right]_{i}=0 .
$$

The computation of the approximate solution to this scheme, on the contrary to the implicit scheme, does not require solving a linear system at each time step (a truncation parameter $m$ is however still needed for the practical implementation), but it is known that the CFL condition, ensuring the $L^{\infty}$-stability of the method, is usually more binding than in the implicit case. Using Formula (3.1), we can make this condition precise: with the same notation as in the proof of Corollary 3.4, and since the index $j=0$ in (3.1) plays no role, the equivalent of (3.10) for the explicit scheme gives

$$
u_{i}^{n+1}=\left(1-a_{i}^{n}-b_{i}^{n}-\delta t \sum_{0<|j| \leq A^{\delta x}} \mu_{j}^{\delta x}\right) u_{i}^{n}+a_{i}^{n} u_{i+1}^{n}+b_{i}^{n} u_{i-1}^{n}+\delta t \sum_{0<|j| \leq A^{\delta x}} \mu_{j}^{\delta x} u_{i+j}^{n} .
$$




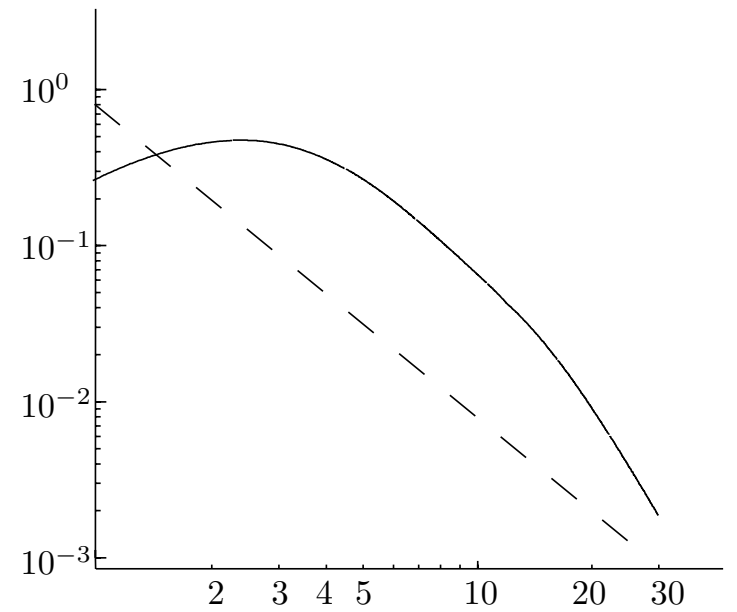

Figure 6. Plot in log-log scale of $t \mapsto \| t^{1 / \lambda}[u(t)-K(t) *$ $\left.u_{0}\right] \|_{L^{\infty}([-1,1])}$ (continuous line) and of $t \mapsto t^{-1 / \lambda}$ (dashed line), for $\lambda=0.5, u_{0}(x)=\mathbf{1}_{[-0.2,0.2]}(x)$ and $u$ a solution to $\partial_{t} u+\partial_{x}\left(u^{2} / 6\right)+$ $g[u]=0$.

A sufficient condition for the $L^{\infty}$-stability of the scheme is that $u_{i}^{n+1}$ is a convex combination of $\left(u_{j}^{n}\right)_{j \in \mathbb{Z}}$, which is, from the definition of $a_{i}^{n}$ and $b_{i}^{n}$, ensured by the preceding relation if

$$
\frac{\delta t}{\delta x}\left(\operatorname{Lip}_{1, u_{0}}(F)+\operatorname{Lip}_{2, u_{0}}(F)\right)+\delta t \sum_{0<|j| \leq A^{\delta x}} \mu_{j}^{\delta x} \leq 1
$$

For the particular example of $g^{\delta x}$ given by (4.3), this comes down to

$$
\frac{\delta t}{\delta x}\left(\operatorname{Lip}_{1, u_{0}}(F)+\operatorname{Lip}_{2, u_{0}}(F)\right)+\frac{\delta t}{(\delta x)^{\lambda}} c(\lambda)\left(\sum_{0<|j| \leq J_{\delta x}} \frac{1}{|j|^{1+\lambda}}+\frac{2}{\lambda J_{\delta x}^{\lambda}}\right) \leq 1
$$

This condition on the time and space steps is more restrictive than (2.4), but in general not terribly more since $c(\lambda)$ is small $(c(0.5) \approx 0.08, c(1) \approx 0.05, c(1.5) \approx$ $0.02)$; this is especially true if $\lambda<1$ : asymptotically as the space step tends to 0 , the term coming from the hyperbolic part of the equation is then leading in (4.7). This is however the opposite if $\lambda>1$, and this CFL condition is also very sensitive to the ratio diffusion/hyperbolic flux: if the hyperbolic flux is smaller than the diffusion term (e.g. if $\operatorname{Lip}_{u_{0}}(f)$ is small, which entails in general that $\operatorname{Lip}_{1, u_{0}}(F)+\operatorname{Lip}_{2, u_{0}}(F)$ is also small, or if we multiply $g$ in (1.1) by a coefficient), (4.7) can be much more demanding than (2.4); at the level of discretization used in the preceding tests and for $\lambda>1$, a ratio of 5 between the coefficient of $g$ and $\operatorname{Lip}_{u_{0}}(f)$ is enough to find a noticeable difference between these two CFL conditions (recall also that the $g$ we used is in fact $\left.g=(2 \pi)^{-\lambda}(-\Delta)^{\lambda / 2}\right)$.

From a practical point of view, if the parameters are chosen so that the explicit scheme is stable (in which case the implicit scheme is of course also stable), the 
solutions given by both forms (explicit and implicit) of the scheme are very similar: for example, in the preceding numerical tests, the relative $L^{\infty}$-norm of their difference is lower than $3 \times 10^{-4}$.

Since the explicit scheme demands to compute $g^{\delta x}\left[u^{n}\right]$ at each time step, its implementation can take advantage of FFT-based algorithms as in Section 4.1.3. In fact, as explained in this section, the implicit scheme also requires such a computation at each time step, and the difference of cost between the explicit and implicit methods therefore lies in the preconditioned CG iterations needed to solve the system in the implicit case. Although very fast, these CG iterations are not negligible in the overall cost and, in situations where (4.7) is not much more demanding than (2.4), the explicit scheme is clearly faster than the implicit scheme. However, for a diffusion-dominated problem (for example $f(s)=s^{2} / 2, \lambda=1.5$ and $(2 \pi)^{\lambda} g=(-\Delta)^{\lambda / 2}$ instead of $\left.g\right)$, (4.7) can impose a much smaller time step than (2.4) and the implicit method then remains way more efficient than the explicit method.

\section{APPENDIX: TECHNICAL LEMMAS}

Lemma 5.1. If $J_{\delta x}$ is such that $J_{\delta x} \delta x \rightarrow+\infty$ as $\delta x \rightarrow 0$, then $g^{\delta x}$ defined by (4.3) satisfies (2.9) and (2.10).

Proof of Lemma [5.1.

Step 1: Proof of (2.9).

We notice first, from (4.3), that $\left(g^{\delta x}\right)^{*}=g^{\delta x}$. Let $K$ be a compact subset of $\mathbb{R}$ and define $A^{\delta x}: C_{K}^{2}(\mathbb{R}) \rightarrow L^{1}(\mathbb{R})$ by $A^{\delta x} \phi=g^{\delta x}[\Phi]-g[\phi]$, where $\Phi$ is defined from $\phi$ as before (2.9) . Proving (2.9) is equivalent to proving that $A^{\delta x} \rightarrow 0$ in $\mathcal{L}\left(C_{K}^{2}(\mathbb{R}) ; L^{1}(\mathbb{R})\right)$ as $\delta x \rightarrow 0$, which we intend to do by applying Lemma 5.3 (stated after this proof).

Let $r>0$ and $x \in \mathbb{R}$; by definition, choosing $i \in \mathbb{Z}$ such that $x \in[i \delta x,(i+1) \delta x[$ we have

$$
\begin{aligned}
g^{\delta x}[\Phi](x)= & -c(\lambda) \sum_{0<|j| \leq r / \delta x} \delta x \frac{\Phi_{i+j}-\Phi_{i}}{|j \delta x|^{1+\lambda}}-c(\lambda) \sum_{r / \delta x<|j| \leq J_{\delta x}} \delta x \frac{\Phi_{i+j}-\Phi_{i}}{|j \delta x|^{1+\lambda}} \\
& -c(\lambda) \frac{\Phi_{i-J_{\delta x}-1}-\Phi_{i}}{\lambda\left(J_{\delta x} \delta x\right)^{\lambda}}-c(\lambda) \frac{\Phi_{i+J_{\delta x}+1}-\Phi_{i}}{\lambda\left(J_{\delta x} \delta x\right)^{\lambda}} \\
= & -c(\lambda) \sum_{0<|j| \leq r / \delta x} \frac{\delta x}{|j \delta x|^{1+\lambda}} \frac{1}{\delta x} \int_{i \delta x}^{(i+1) \delta x} \phi(\xi+j \delta x)-\phi(\xi) d \xi \\
& -c(\lambda) \sum_{r / \delta x<|j| \leq J_{\delta x}} \frac{\delta x}{|j \delta x|^{1+\lambda} \frac{1}{\delta x} \int_{i \delta x}^{(i+1) \delta x} \phi(\xi+j \delta x)-\phi(\xi) d \xi} \\
& -c(\lambda) \frac{1}{\lambda\left(J_{\delta x} \delta x\right)^{\lambda}} \frac{1}{\delta x}\left(\int_{\left(i-J_{\delta x}-1\right) \delta x}^{\left(i-J_{\delta x}\right) \delta x} \phi(\xi) d \xi-\int_{i \delta x}^{(i+1) \delta x} \phi(\xi) d \xi\right) \\
& -c(\lambda) \frac{1}{\lambda\left(J_{\delta x} \delta x\right)^{\lambda}} \frac{1}{\delta x}\left(\int_{\left(i+J_{\delta x}+1\right) \delta x}^{\left(i+J_{\delta x}+2\right) \delta x} \phi(\xi) d \xi-\int_{i \delta x}^{(i+1) \delta x} \phi(\xi) d \xi\right) .
\end{aligned}
$$

\footnotetext{
${ }^{3}$ It might not be straightforward that $A^{\delta x}$ takes its values in $L^{1}(\mathbb{R})$, because of the term $g^{\delta x}[\Phi]$, but this will be made clear by the reasoning to come.
} 
The same way we went from (4.1) to (4.2), we can add to each term in the first sum of the right-hand side anything of the form $p_{i} \frac{j \delta x}{|j \delta x|^{1+\lambda}}$ without changing the value of the sum (these additional terms cancel out each other by symmetry). We choose to add $-\int_{i \delta x}^{(i+1) \delta x} \phi^{\prime}(\xi) d \xi \frac{j \delta x}{|j \delta x|^{1+\lambda}}$ and we obtain

$$
\begin{aligned}
g^{\delta x}[\Phi](x)= & -c(\lambda) \sum_{0<|j| \leq r / \delta x} \frac{\delta x}{|j \delta x|^{1+\lambda}} \frac{1}{\delta x} \int_{i \delta x}^{(i+1) \delta x} \phi(\xi+j \delta x)-\phi(\xi)-\phi^{\prime}(\xi) j \delta x d \xi \\
& -c(\lambda) \sum_{r / \delta x<|j| \leq J_{\delta x}} \frac{\delta x}{|j \delta x|^{1+\lambda}} \frac{1}{\delta x} \int_{i \delta x}^{(i+1) \delta x} \phi(\xi+j \delta x)-\phi(\xi) d \xi \\
& -c(\lambda) \frac{1}{\lambda\left(J_{\delta x} \delta x\right)^{\lambda}} \frac{1}{\delta x}\left(\int_{\left(i-J_{\delta x}-1\right) \delta x}^{\left(i-J_{\delta x}\right) \delta x} \phi(\xi) d \xi-\int_{i \delta x}^{(i+1) \delta x} \phi(\xi) d \xi\right) \\
& -c(\lambda) \frac{1}{\lambda\left(J_{\delta x} \delta x\right)^{\lambda}} \frac{1}{\delta x}\left(\int_{\left(i+J_{\delta x}+1\right) \delta x}^{\left(i+J_{\delta x}+2\right) \delta x} \phi(\xi) d \xi-\int_{i \delta x}^{(i+1) \delta x} \phi(\xi) d \xi\right) \\
= & T_{8}^{\delta x, r}[\phi](x)+T_{9}^{\delta x, r}[\phi](x)+T_{10}^{\delta x}[\phi](x)+T_{11}^{\delta x}[\phi](x) .
\end{aligned}
$$

We then define the operators $A_{0, r}^{\delta x}$ and $A_{2, r}^{\delta x}$ by

$$
A_{0, r}^{\delta x} \phi=T_{0}^{\delta x, r}[\phi]+T_{10}^{\delta x}[\phi]+T_{11}^{\delta x}[\phi]-g_{0, r}[\phi] \quad \text { and } \quad A_{2, r}^{\delta x} \phi=T_{8}^{\delta x, r}[\phi]-g_{\lambda, r}[\phi] .
$$

The definition of $g_{0, r}$ clearly shows that $A_{0, r}^{\delta x} \phi$ is defined for any $\phi \in C_{K}^{0}(\mathbb{R})$. For all $x \in \mathbb{R}$ we have

$$
\begin{aligned}
& \left|T_{\frac{10}{\delta x}[\phi]}[x)+T_{[11]}^{\delta x}[\phi](x)\right| \\
& \leq \frac{c(\lambda)}{\lambda\left(J_{\delta x} \delta x\right)^{\lambda}}\left(\sup _{|s| \leq \delta x}\left|\phi\left(x-J_{\delta x} \delta x-\delta x+s\right)\right|+2 \sup _{|s| \leq \delta x}|\phi(x+s)|\right. \\
& \left.\quad+\sup _{|s| \leq \delta x}\left|\phi\left(x+J_{\delta x} \delta x+\delta x+s\right)\right|\right),
\end{aligned}
$$

which shows, integrating and using some changes of variables, that

$$
\begin{aligned}
\left\|T_{\frac{10}{10}}^{\delta x}[\phi]+T_{[11}^{\delta x}[\phi]\right\|_{L^{1}(\mathbb{R})} & \leq \frac{4 c(\lambda)}{\lambda\left(J_{\delta x} \delta x\right)^{\lambda}} \int_{\mathbb{R}} \sup _{|s| \leq \delta x}|\phi(z+s)| d z \\
& \leq \frac{4 c(\lambda)}{\lambda\left(J_{\delta x} \delta x\right)^{\lambda}}\|\phi\|_{L^{\infty}(\mathbb{R})} \operatorname{mes}(K+[-\delta x, \delta x])
\end{aligned}
$$

and thus, since $J_{\delta x} \delta x \rightarrow \infty$ as $\delta x \rightarrow 0$,

$$
T_{10}^{\delta x}+T_{11}^{\delta x} \rightarrow 0 \quad \text { in } \mathcal{L}\left(C_{K}^{0}(\mathbb{R}) ; L^{1}(\mathbb{R})\right) \text { as } \delta x \rightarrow 0 .
$$

We let $I(j, \delta x)=[j \delta x,(j+1) \delta x[$ if $j>0$ and $I(j, \delta x)=](j-1) \delta x, j \delta x]$ if $j<0$, and we define $H^{\delta x}: \mathbb{R} \rightarrow \mathbb{R}$ by: for all $r / \delta x<|j| \leq J_{\delta x}, H^{\delta x}=\frac{1}{|j \delta x|^{1+\lambda}}$ on $I(j, \delta x)$, and $H^{\delta x}=0$ on $\mathbb{R} \backslash \bigcup_{r / \delta x<|j| \leq J_{\delta x}} I(j, \delta x)$. By regularity of $z \rightarrow|z|^{-1-\lambda}$ on $|z| \geq r$, we have, if $\delta x<r / 2$,

$$
\begin{aligned}
\forall z \in \bigcup_{r / \delta x<|j| \leq J_{\delta x}} I(j, \delta x):\left|H^{\delta x}(z)-\frac{1}{|z|^{1+\lambda} \mid}\right| & \leq(1+\lambda) \delta x \sup _{|s| \leq \delta x} \frac{1}{|z+s|^{2+\lambda}} \\
& \leq \delta x \frac{1+\lambda}{(|z|-r / 2)^{2+\lambda}} .
\end{aligned}
$$


Since $\bigcup_{r / \delta x<|j| \leq J_{\delta x}} I(j, \delta x)=\left\{z \in \mathbb{R}\left|r+\alpha_{r, \delta x} \delta x \leq\right| z \mid<J_{\delta x} \delta x+\delta x\right\}$ for some $\alpha_{r, \delta x} \in[0,1]$, we deduce that $H^{\delta x} \rightarrow \frac{1}{|z|^{1+\lambda}}$ in $L^{1}(|z|>r)$ as $\delta x \rightarrow 0$; for all $\phi \in C_{K}^{0}(\mathbb{R})$ and all $x \in \mathbb{R}$, by uniform continuity of $\phi$ we infer that

$$
\mathrm{T}_{[9}^{\delta x, r}[\phi](x) \rightarrow-c(\lambda) \int_{|z|>r} \frac{\phi(x+z)-\phi(x)}{|z|^{1+\lambda}} d z=g_{0, r}[\phi](x) \quad \text { as } \delta x \rightarrow 0 .
$$

Moreover, still assuming that $\delta x<r / 2$,

$$
\left|T_{\square}^{\delta x, r}[\phi](x)\right| \leq c(\lambda) \int_{|z|>r} H^{\delta x}(z)\left(\sup _{|s| \leq r}|\phi(x+z+s)|+\sup _{|s| \leq r / 2}|\phi(x+s)|\right) d z,
$$

and, by convergence of $H^{\delta x}$ in $L^{1}(|z|>r)$, the right-hand side of (5.3) converges, as a function of $x$, in $L^{1}(\mathbb{R})$ as $\delta x \rightarrow 0$. The dominated convergence theorem and (5.2) then show that $T_{9}^{\delta x, r}[\phi] \rightarrow g_{0, r}[\phi]$ in $L^{1}(\mathbb{R})$ and, together with (5.1), this proves that $A_{0, r}^{\delta x}$ satisfies Item 1 in Lemma 5.3 .

Since $\left(H^{\delta x}\right)_{\delta x \in] 0, r / 2[}$ is bounded in $L^{1}(|z|>r)$, (5.3) gives $\| T_{9}^{\delta x, r}[\phi]||_{L^{1}(\mathbb{R})} \leq$

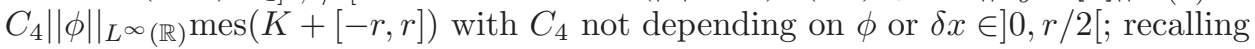
(5.1) and since $g_{0, r} \in \mathcal{L}\left(C_{K}^{0}(\mathbb{R}) ; L^{1}(\mathbb{R})\right)$ (see the definition of $g_{0, r}$ ), this shows that $A_{0, r}^{\delta x}$ satisfies Item 2 in Lemma 5.3 .

Let us now turn to $A_{2, r}^{\delta x}$. Writing $\phi(x+z)-\phi(x)-\phi^{\prime}(x) z=\int_{0}^{1}(1-s) \phi^{\prime \prime}(x+$ $s z) z^{2} d s$, we have

$$
\left\|g_{\lambda, r}[\phi]\right\|_{L^{1}(\mathbb{R})} \leq c(\lambda)\left\|\phi^{\prime \prime}\right\|_{L^{1}(\mathbb{R})} \int_{|z| \leq r}|z|^{1-\lambda} d z \leq\left\|\phi^{\prime \prime}\right\|_{L^{1}(\mathbb{R})} \frac{2 c(\lambda)}{2-\lambda} r^{2-\lambda} .
$$

We handle $T_{[8}^{\delta x, r}[\phi]$ in a similar way: integrating its definition with respect to $x$ and using a comparison between discrete and integral sums, we find that

$$
\begin{aligned}
\left\|T_{\mathbb{8}}^{\delta x, r}[\phi](x)\right\|_{L^{1}(\mathbb{R})} & \leq c(\lambda) \sum_{0<|j| \leq r / \delta x} \frac{\delta x}{|j \delta x|^{1+\lambda}} \int_{\mathbb{R}}\left|\phi(\xi+j \delta x)-\phi(\xi)-\phi^{\prime}(\xi) j \delta x\right| d \xi \\
& \leq c(\lambda) \sum_{0<|j| \leq r / \delta x} \frac{\delta x}{|j \delta x|^{1+\lambda}}\left\|\phi^{\prime \prime}\right\|_{L^{1}(\mathbb{R})}|j \delta x|^{2} \\
& \leq\left\|\phi^{\prime \prime}\right\|_{L^{1}(\mathbb{R})} c(\lambda) \int_{|z| \leq r+\delta x}|z|^{1-\lambda} d z \\
& \leq\left\|\phi^{\prime \prime}\right\|_{L^{1}(\mathbb{R})} \frac{2 c(\lambda)}{2-\lambda}(r+\delta x)^{2-\lambda} .
\end{aligned}
$$

Together with (5.4), this proves that $A_{2, r}^{\delta x}$ satisfies Item 3 in Lemma 5.3 and concludes the proof of (2.9).

Step 2: Proof of (2.10).

The cutting of $g^{\delta x}$ in $g_{\lambda, r}^{\delta x}$ and $g_{0, r}^{\delta x}$ is of course the one given by (4.2), $g_{\lambda, r}^{\delta x}$ being the first sum and $g_{0, r}^{\delta x}$ the rest of the right-hand side. The proof that $g_{\lambda, r}^{\delta x}$ satisfies (2.5) -(2.8) and (2.9) with $g_{\lambda, r}$ instead of $g$ is done exactly as for $g^{\delta x}$ (the proof of (2.9) is done by cutting the sum defining $g_{\lambda, r}^{\delta x}$ at a level $r^{\prime} / \delta x$ with $r^{\prime}<r$, by introducing the derivative of $\phi$ in the lower part of the sum and by replacing, in the reasoning of Step 1 above and in Lemma 5.3, $r$ with $r^{\prime}$ ). 
Let us study $g_{0, r}^{\delta x}$. For all $v \in l^{\infty}(\mathbb{Z})$ and all $x \in \mathbb{R}$, choosing $i \in \mathbb{Z}$ such that $x \in[i \delta x,(i+1) \delta x[$ we have

$g_{0, r}^{\delta x}[v](x)=-c(\lambda) \sum_{r / \delta x<|j| \leq J_{\delta x}} \delta x \frac{v_{i+j}-v_{i}}{|j \delta x|^{1+\lambda}}-c(\lambda) \frac{v_{i-J_{\delta x}-1}-v_{i}}{\lambda\left(J_{\delta x} \delta x\right)^{\lambda}}-c(\lambda) \frac{v_{i+J_{\delta x}+1}-v_{i}}{\lambda\left(J_{\delta x} \delta x\right)^{\lambda}}$.

Let $|j|>r / \delta x$. We have $x+z \in[(i+j) \delta x,(i+j+1) \delta x[$ (which implies $v(x+$ $\left.z)=v_{i+j}\right)$ if and only if $z \in\left[j \delta x,(j+1) \delta x\left[+(i \delta x-x)=: E_{j}(i, x)\right.\right.$, in which case $|z-j \delta x| \leq \delta x$ and, if $\delta x \leq r / 4$,

$$
\left|\frac{1}{|z|^{1+\lambda}}-\frac{1}{|j \delta x|^{1+\lambda}}\right| \leq(1+\lambda) \delta x \sup _{|s| \leq \delta x} \frac{1}{|z+s|^{2+\lambda}} \leq \delta x(1+\lambda) \frac{1}{(|z|-r / 4)^{2+\lambda}}
$$

(notice that if $z \in E_{j}(i, x)$, then $|z|>r / 2$ ). We deduce

$$
\begin{aligned}
\mid \delta x \frac{v_{i+j}-v_{i}}{|j \delta x|^{1+\lambda}} & -\int_{E_{j}(i, x)} \frac{v(x+z)-v(x)}{|z|^{1+\lambda}} d z \mid \\
& \leq 2\|v\|_{l^{\infty}(\mathbb{Z})} \delta x(1+\lambda) \int_{E_{j}(i, x)} \frac{d z}{(|z|-r / 4)^{2+\lambda}}
\end{aligned}
$$

and, plugging this into (5.5) and defining $E(i, x)=\bigcup_{r / \delta x<|j| \leq J_{\delta x}} E_{j}(i, x) \subset\{|z|>$ $r / 2\}$,

$$
\begin{aligned}
\left|g_{0, r}^{\delta x}[v](x)+c(\lambda) \int_{E(i, x)} \frac{v(x+z)-v(x)}{|z|^{1+\lambda}} d z\right| \leq & 2\|v\|_{l^{\infty}(\mathbb{Z})} \delta x(1+\lambda) \int_{|z|>r / 2} \frac{d z}{(|z|-r / 4)^{2+\lambda}} \\
& +\frac{4 c(\lambda)\|v\|_{l^{\infty}(\mathbb{Z})}}{\lambda\left(J_{\delta x} \delta x\right)^{\lambda}} .
\end{aligned}
$$

But $E(i, x)=\left[-J_{\delta x} \delta x+\rho_{1}^{\prime} \delta x,-r+\rho_{1} \delta x\left[\cup\left[r+\rho_{2} \delta x, J_{\delta x} \delta x+\rho_{2}^{\prime} \delta x\left[\right.\right.\right.\right.$ with $\left(\rho_{1}, \rho_{1}^{\prime}, \rho_{2}, \rho_{2}^{\prime}\right)$ $\in[-1,1]$ and the symmetric difference between $E(i, x)$ and $\{|z|>r\}$ is therefore contained in $\{r-\delta x \leq|z| \leq r+\delta x\} \cup\left\{|z| \geq J_{\delta x} \delta x-\delta x\right\}$; we conclude that

$$
\begin{aligned}
& \left|g_{0, r}^{\delta x}[v](x)-g_{0, r}\left[v_{\delta x}\right](x)\right| \\
& \leq \quad 2\|v\|_{l^{\infty}(\mathbb{Z})} \delta x(1+\lambda) \int_{|z|>r / 2} \frac{d z}{(|z|-r / 4)^{2+\lambda}}+\frac{4 c(\lambda)\|v\|_{l^{\infty}(\mathbb{Z})}}{\lambda\left(J_{\delta x} \delta x\right)^{\lambda}} \\
& \quad+2 c(\lambda)\|v\|_{l^{\infty}(\mathbb{Z})} \int_{r-\delta x \leq|z| \leq r+\delta x} \frac{1}{|z|^{1+\lambda}} d z \\
& \quad+2\|v\|_{l^{\infty}(\mathbb{Z})} c(\lambda) \int_{|z|>J_{\delta x} \delta x-\delta x} \frac{1}{|z|^{1+\lambda}} d z
\end{aligned}
$$

and Item 2 of (2.10) follows, the estimate being in fact valid in $L^{\infty}(\mathbb{R})$ and not only in $L^{1}(Q)$.

Remark 5.2. It is also possible, by some direct estimates rather than using the abstract Lemma 5.3. to give an explicit $\theta_{K}$ such that (2.9) holds; such an expression could be useful, for example, to establish error estimates for the scheme (2.1)-(2.2). However, getting this $\theta_{K}$ is much more technical than the arguments used in Step 1 of the preceding proof. 
Lemma 5.3. Let $K$ be a compact subset of $\mathbb{R}$ and denote by $C_{K}^{0}(\mathbb{R})$ (resp. $C_{K}^{2}(\mathbb{R})$ ) the space of continuous (resp. twice continuously differentiable) functions with support included in $K$. Assume that $\left(A^{\delta x}\right)_{\delta x>0}$ is a family of linear continuous operators $C_{K}^{2}(\mathbb{R}) \rightarrow L^{1}(\mathbb{R})$ such that, for all $r>0$, we can write $A^{\delta x}=A_{0, r}^{\delta x}+A_{2, r}^{\delta x}$ with $A_{0, r}^{\delta x}: C_{K}^{0}(\mathbb{R}) \rightarrow L^{1}(\mathbb{R})$ and $A_{2, r}^{\delta x}: C_{K}^{2}(\mathbb{R}) \rightarrow L^{1}(\mathbb{R})$ linear continuous operators satisfying:

(1) for all $r>0$ and all $\phi \in C_{K}^{0}(\mathbb{R}), A_{0, r}^{\delta x} \phi \rightarrow 0$ in $L^{1}(\mathbb{R})$ as $\delta x \rightarrow 0$,

(2) for all $r>0$, $\lim \sup _{\delta x \rightarrow 0}\left\|A_{0, r}^{\delta x}\right\|_{\mathcal{L}\left(C_{K}^{0}(\mathbb{R}) ; L^{1}(\mathbb{R})\right)}<+\infty$,

(3) $\lim _{r \rightarrow 0} \lim \sup _{\delta x \rightarrow 0}\left\|A_{2, r}^{\delta x}\right\|_{\mathcal{L}\left(C_{K}^{2}(\mathbb{R}) ; L^{1}(\mathbb{R})\right)}=0$.

Then $A^{\delta x} \rightarrow 0$ in $\mathcal{L}\left(C_{K}^{2}(\mathbb{R}) ; L^{1}(\mathbb{R})\right)$ as $\delta x \rightarrow 0$.

Proof of Lemma 5.3. We take $\phi^{\delta x} \in C_{K}^{2}(\mathbb{R})$ such that $\left\|\phi^{\delta x}\right\|_{C_{K}^{2}(\mathbb{R})} \leq 1$ and $\left\|A^{\delta x}\right\|_{\mathcal{L}\left(C_{K}^{2}(\mathbb{R}) ; L^{1}(\mathbb{R})\right)} \leq\left\|A^{\delta x} \phi^{\delta x}\right\|_{L^{1}(\mathbb{R})}+\delta x$. Using the compactness of the embedding $C_{K}^{2}(\mathbb{R}) \hookrightarrow C_{K}^{0}(\mathbb{R})$, we can assume that $\phi^{\delta x}$ converges in $C_{K}^{0}(\mathbb{R})$ to some $\phi$ as $\delta x \rightarrow 0$. We then take $r>0$ and write

$$
\begin{aligned}
\left\|A^{\delta x}\right\|_{\mathcal{L}\left(C_{K}^{2}(\mathbb{R}) ; L^{1}(\mathbb{R})\right)} \leq & \left\|A_{0, r}^{\delta x} \phi^{\delta x}\right\|_{L^{1}(\mathbb{R})}+\left\|A_{2, r}^{\delta x} \phi^{\delta x}\right\|_{L^{1}(\mathbb{R})}+\delta x \\
\leq & \left\|A_{0, r}^{\delta x} \phi\right\|_{L^{1}(\mathbb{R})}+\left\|A_{0, r}^{\delta x}\right\|_{\mathcal{L}\left(C_{K}^{0}(\mathbb{R}) ; L^{1}(\mathbb{R})\right)}\left\|\phi^{\delta x}-\phi\right\|_{C_{K}^{0}(\mathbb{R})} \\
& +\left\|A_{2, r}^{\delta x}\right\|_{\mathcal{L}\left(C_{K}^{2}(\mathbb{R}) ; L^{1}(\mathbb{R})\right)}+\delta x,
\end{aligned}
$$

and the conclusion follows by taking first the upper limit as $\delta x \rightarrow 0$ and then the limit as $r \rightarrow 0$.

\section{ACKNOWLEDGEMENTS}

The author would like to thank the referee for his/her useful suggestions, which have led to a reduction of technicality in some reasonings (in particular in Step 1 of the proof of Lemma 5.1).

\section{REFERENCES}

[1] Alibaud N., Entropy formulation for fractal conservation laws, J. Evol. Equ., 7 (2007), no. 1, 145-175. MR2305729(2009d:35213)

[2] Alibaud N., Azerad P. And Isebe D., A non-monotone nonlocal conservation law for dune morphodynamics, submitted for publication.

[3] Alibaud N., Droniou J. and Vovelle J., Occurrence and non-appearance of shocks in fractal Burgers equations, J. Hyperbolic Differ. Equ. 4 (2007), no. 3, 479-499. MR 2339805 (2008i:35198)

[4] Aubin T., Un théorème de compacité, C.R. Acad. Sci. Paris, 256 (1963), 5042-5044. MR 0152860(27:2832)

[5] Alvarez O., Hoch P., Le Bouar Y. and Monneau R., Dislocation dynamics: Short-time existence and uniqueness of the solution, Arch. Ration. Mech. Anal. 181 (2006), no. 3, 449504. MR2231781 (2007d:74019)

[6] Biler P., Funaki T. And Woyczyński W., Fractal Burgers Equations, J. Diff. Eq., 148 (1998), 9-46. MR:1637513 (99g:35111)

[7] Biler P., Karch G. AND WoyczyŃski W., Asymptotics for conservation laws involving Lévy diffusion generators, Studia Mathematica 148 (2001), no. 2, 171-192. MR 1881259 (2002j:60140)

[8] Biler P., Karch G. and Woyczyński W., Critical nonlinearity exponent and self-similar asymptotics for Lévy conservation laws, Ann. Inst. H. Poincaré Anal. Non Linéaire 18 (2001), no. 5, 613-637. MR1849690 (2002f:35035)

[9] Bony J-M., Courrège P., Priouret P., Semi-groupes de Feller sur une variété à bord compacte et problèmes aux limites intégro-différentiels du second ordre donnant lieu au principe du maximum, Ann. Inst. Fourier (Grenoble), 18 (1968), no. 2, 369-521. MR0245085(39:6397) 
[10] Eymard R., Gallouët T., Herbin R., Finite Volume Methods, Handbook of Numerical Analysis, Vol. VII Edited by P.G. Ciarlet and J.L. Lions, North-Holland, 713-1020 (2000). MR:1804748 (2002e:65138)

[11] Chainais-Hillairet C. And Droniou J., Convergence analysis of a mixed finite volume scheme for an elliptic-parabolic system modelling miscible fluid flows in porous media, SIAM J. Numer. Anal. 45 (2007), no. 5, 2228-2258. MR2346377(2008i:76129)

[12] Chan R.H. AND NG M.K., Conjugate gradient methods for Toeplitz systems. SIAM Review 38 (3), 1996, 427-482. MR1409592 (97i:65048)

[13] Clavin P., Instabilities and nonlinear patterns of overdriven detonations in gases, $\mathrm{H}$. Berestycki and Y. Pomeau (eds.), Nonlinear PDE's in Condensed Matter and Reactive Flows, Kluwer (2002), 49-97.

[14] Droniou J., Gallouët T. And Vovelle J., Global solution and smoothing effect for a non-local regularization of a hyperbolic equation, J. Evol. Equ. 3 (2003), no. 3, 499-521. MR2019032 (2004m:35168)

[15] Droniou, J. And Imbert, C., Fractal first-order partial differential equations, Arch. Ration. Mech. Anal. 182 (2006), no. 2, 299-331. MR2259335 (2009c:35037)

[16] Ghorbel A. And Monneau R., Well-posedness and numerical analysis of a one-dimensional non-local transport equation modelling dislocations dynamics, submitted for publication.

[17] Godlewski E. and Raviart P.A., Numerical approximation of hyperbolic systems of conservation laws, Applied Math. Sciences 118, Springer, New-York, 1996. MR 1410987(98d:65109)

[18] Krushrov S.N., First order quasilinear equations with several independent variables, Math. Sb. (N.S.) 81 (1970), no. 123, 228-255. MR0267257(42:2159)

[19] Jourdain B., Probabilistic Interpretation via Spatial Derivation of Some Nonlinear Parabolic Evolution Equations, Monte Carlo and Quasi-Monte Carlo Methods 2004, H. Niederreiter and D. Talay (Eds.), Springer-Verlag 2006, pp. 197-216 MR2208710(2007h:65010)

[20] Jourdain B., MélÉard S. and Woyczynski W., Probabilistic approximation and inviscid limits for one-dimensional fractional conservation laws, Bernoulli 11 (2005), no. 4, 689-714. MR2158256 (2006e:60094)

[21] Simon J., Compact sets in the space $L^{p}(0, T ; B)$, Ann. Mat. Pura Appl. (IV), 146 (1987), 65-96 MR 916688 (89c:46055)

[22] Salapaka S., Peirce A. And Dahleh M., Analysis of a circulant based preconditioner for a class of lower rank extracted systems, Numer. Linear Algebra Appl. 12 (2005), no. 1, 9-32. MR2114602 (2005k:65072)

[23] Soner H.M., Optimal control with state-space constraint. II, SIAM J. Control Optim. 24 (1986), no. 6, 1110-1122. MR861089 (87k:49021)

[24] Stanescu D., Kim D. and Woyczynski W., Numerical study of interacting particles approximation for integro-differential equations, Journal of Computational Physics 206 (2005), 706-726. MR2143331 (2006b:65010)

[25] VAn Loan C., Computational frameworks for the fast Fourier transform, Frontiers in Applied Mathematics, 10. Society for Industrial and Applied Mathematics (SIAM). Philadelphia, PA, 1992. MR1153025 (93a:65186)

[26] WOYCZYŃSKI W.A., Lévy processes in the physical sciences, Lévy processes, 241-266, Birkhäuser Boston, Boston, MA, 2001. MR1833700(2002d:82029)

Université Montpellier 2, Institut de Mathématiques et de Modélisation de MontPellier, CC 051, Place Eugène Bataillon, 34095 Montpellier cedex 5, France

E-mail address: droniou@math.univ-montp2.fr 